\title{
Determinantal representations of the Moore-Penrose inverse over the quaternion skew field and corresponding Cramer's rules.
}

\author{
Ivan Kyrchei*
}

\begin{abstract}
Determinantal representation of the Moore-Penrose inverse over the quaternion skew field is obtained within the framework of a theory of the column and row determinants. Using the obtained analogs of the adjoint matrix, we get the Cramer rules for the least squares solution of left and right systems of quaternionic linear equations.
\end{abstract}

Keywords: Moore-Penrose inverse, quaternion matrix, least square solution, Cramer rule, quaternionic linear equation.

AMS classification:15A06,15A09, 15A15, 15A33

\section{Introduction}

The existence, uniqueness and full-rank representation of the Moore-Penrose inverse over the quaternion skew field $\mathbb{H}$ have been studied in particular in [10, 22, 19]. At the same time the problem of determinantal representation of the Moore-Penrose inverse of a quaternion matrix is still unsolved. Moreover, by means of any introduced determinants currently it is not possible to receive a determinantal representation of an inverse matrix over $\mathbb{H}$. The problem consists in the fact that there is no determinant functional over a skew field which would keep all the properties inherent in the complex case, as is proved in [1]. So the determinants of Dieudonne [5] and Study [16] assume values

*Pidstrygach Institute for Applied Problems of Mechanics and Mathematics, str.Naukova 3b, Lviv, Ukraine, 79005, kyrchei@lms.lviv.ua 
not in a skew field, but in the field which is its center. These determinants can not be expanded by cofactors along an arbitrary row or column of a matrix. The Moore determinant is introduced [14] in terms of permutations only on the class of quaternion Hermitian matrices. The other determinant in terms of permutations, the determinant of L. Chen [4] dissatisfies a key property of a determinant, its singularity for noninvertible matrices. The double determinant introduced by L. Chen can not be expanded by cofactors along an arbitrary row or column of a matrix as well.

In this paper determinantal representations of the Moore-Penrose inverse are obtained within the framework of the theory of new matrix functionals over the quaternion skew field (the column and row determinants) introduced in [11. In the first point we cite some provisions from the theory of the column and row determinants which are necessary for the following. The theory of the column and row determinants of a quaternionic matrix is considered completely in [11]. In the second point some known facts from the theory of eigenvalues of a quaternion matrix and its singular value decomposition are considered. The concept of a characteristic polynomial of a Hermitian matrix is introduced and its coefficients are investigated. A Moore-Penrose inverse over a quaternion skew field and its limit representation are introduced in this point as well. In the third point the theorems about determinantal representations of the Moore-Penrose inverse and the projection matrices $\mathbf{A}^{+} \mathbf{A}$, $\mathbf{A A}^{+}$for an arbitrary matrix $\mathbf{A} \in \mathbb{H}^{m \times n}$ are proved. In the fourth point the Cramer rules for a least squares solutions of right and left systems of linear equations over a quaternion skew field are obtained.

\section{Elements of the theory of the column and row determinants.}

Let $\mathrm{M}(n, \mathbb{H})$ be the ring of $n \times n$ quaternion matrices. By $\mathbb{H}^{m \times n}$ denote the set of all $m \times n$ matrices over the quaternion skew field $\mathbb{H}$ and by $\mathbb{H}_{r}^{m \times n}$ denote its subset of matrices of rank $r$. Suppose $S_{n}$ is the symmetric group on the set $I_{n}=\{1, \ldots, n\}$.

Definition 2.1 The ith row determinant of $\mathbf{A}=\left(a_{i j}\right) \in \mathrm{M}(n, \mathbb{H})$ is defined by 


$$
\operatorname{rdet}_{i} \mathbf{A}=\sum_{\sigma \in S_{n}}(-1)^{n-r} a_{i i_{k_{1}}} a_{i_{k_{1}} i_{k_{1}+1}} \ldots a_{i_{k_{1}+l_{1}} i} \ldots a_{i_{k_{r}} i_{k_{r}+1}} \ldots a_{i_{k_{r}+l_{r}} i_{k_{r}}}
$$

for all $i=1, \ldots, n$. The elements of the permutation $\sigma$ are indices of each monomial. The left-ordered cycle notation of the permutation $\sigma$ is written as follows,

$$
\sigma=\left(i i_{k_{1}} i_{k_{1}+1} \ldots i_{k_{1}+l_{1}}\right)\left(i_{k_{2}} i_{k_{2}+1} \ldots i_{k_{2}+l_{2}}\right) \ldots\left(i_{k_{r}} i_{k_{r}+1} \ldots i_{k_{r}+l_{r}}\right) .
$$

The index $i$ opens the first cycle from the left and other cycles satisfy the following conditions, $i_{k_{2}}<i_{k_{3}}<\ldots<i_{k_{r}}$ and $i_{k_{t}}<i_{k_{t}+s}$ for all $t=2, \ldots, r$ and $s=1, \ldots, l_{t}$.

Definition 2.2 The jth column determinant of $\mathbf{A}=\left(a_{i j}\right) \in \mathrm{M}(n, \mathbb{H})$ is defined by

$$
\operatorname{cdet}_{j} \mathbf{A}=\sum_{\tau \in S_{n}}(-1)^{n-r} a_{j_{k_{r}} j_{k_{r}+l_{r}}} \ldots a_{j_{k_{r}+1} i_{k_{r}}} \ldots a_{j j_{k_{1}+l_{1}}} \ldots a_{j_{k_{1}+1} j_{k_{1}}} a_{j_{k_{1}} j}
$$

for all $j=1, \ldots, n$. The right-ordered cycle notation of the permutation $\tau \in S_{n}$ is written as follows,

$$
\tau=\left(j_{k_{r}+l_{r}} \ldots j_{k_{r}+1} j_{k_{r}}\right) \ldots\left(j_{k_{2}+l_{2}} \ldots j_{k_{2}+1} j_{k_{2}}\right)\left(j_{k_{1}+l_{1}} \ldots j_{k_{1}+1} j_{k_{1}} j\right)
$$

The index $j$ opens the first cycle from the right and other cycles satisfy the following conditions, $j_{k_{2}}<j_{k_{3}}<\ldots<j_{k_{r}}$ and $j_{k_{t}}<j_{k_{t}+s}$ for all $t=2, \ldots, r$ and $s=1, \ldots, l_{t}$.

Suppose $\mathbf{A}^{i j}$ denotes the submatrix of $\mathbf{A}$ obtained by deleting both the $i$ th row and the $j$ th column. Let $\mathbf{a}_{j}$ be the $j$ th column and $\mathbf{a}_{i \text {. be the } i \text { th }}$ row of $\mathbf{A}$. Suppose $\mathbf{A}_{. j}$ (b) denotes the matrix obtained from $\mathbf{A}$ by replacing its $j$ th column with the column $\mathbf{b}$, and $\mathbf{A}_{i \text {. }}$ (b) denotes the matrix obtained from $\mathbf{A}$ by replacing its $i$ th row with the row $\mathbf{b}$.

We note some properties of column and row determinants of a quaternion matrix $\mathbf{A}=\left(a_{i j}\right)$, where $i \in I_{n}, j \in J_{n}$ and $I_{n}=J_{n}=\{1, \ldots, n\}$.

Proposition 2.1 [11] If $b \in \mathbb{H}$, then $\operatorname{rdet}_{i} \mathbf{A}_{i .}\left(b \cdot \mathbf{a}_{i .}\right)=b \cdot \operatorname{rdet}_{i} \mathbf{A}$ for all $i=1, \ldots, n$. 
Proposition 2.2 [11] If $b \in \mathbb{H}$, then $\operatorname{cdet}_{j} \mathbf{A}_{. j}\left(\mathbf{a}_{. j} b\right)=\operatorname{cdet}_{j} \mathbf{A} b$ for all $j=$ $1, \ldots, n$.

Proposition 2.3 [11] If for $\mathbf{A} \in \mathrm{M}(n, \mathbb{H})$ there exists $t \in I_{n}$ such that $a_{t j}=b_{j}+c_{j}$ for all $j=1, \ldots, n$, then

$$
\begin{aligned}
\operatorname{rdet}_{i} \mathbf{A} & =\operatorname{rdet}_{i} \mathbf{A}_{t .}(\mathbf{b})+\operatorname{rdet}_{i} \mathbf{A}_{t .}(\mathbf{c}), \\
\operatorname{cdet}_{i} \mathbf{A} & =\operatorname{cdet}_{i} \mathbf{A}_{t .}(\mathbf{b})+\operatorname{cdet}_{i} \mathbf{A}_{t .}(\mathbf{c}),
\end{aligned}
$$

where $\mathbf{b}=\left(b_{1}, \ldots, b_{n}\right), \mathbf{c}=\left(c_{1}, \ldots, c_{n}\right)$ and for all $i=1, \ldots, n$.

Proposition 2.4 [11] If for $\mathbf{A} \in \mathrm{M}(n, \mathbb{H})$ there exists $t \in J_{n}$ such that $a_{i t}=b_{i}+c_{i}$ for all $i=1, \ldots, n$, then

$$
\begin{aligned}
& \operatorname{rdet}_{j} \mathbf{A}=\operatorname{rdet}_{j} \mathbf{A} \cdot t(\mathbf{b})+\operatorname{rdet}_{j} \mathbf{A} \cdot t(\mathbf{c}), \\
& \operatorname{cdet}_{j} \mathbf{A}=\operatorname{cdet}_{j} \mathbf{A} \cdot t(\mathbf{b})+\operatorname{det}_{j} \mathbf{A} \cdot t(\mathbf{c}),
\end{aligned}
$$

where $\mathbf{b}=\left(b_{1}, \ldots, b_{n}\right)^{T}, \mathbf{c}=\left(c_{1}, \ldots, c_{n}\right)^{T}$ and for all $j=1, \ldots, n$.

The following lemmas enable us to expand $\operatorname{rdet}_{i} \mathbf{A}$ by cofactors along the $i$ th row and $\operatorname{cdet}_{j} \mathbf{A}$ along the $j$ th column respectively for all $i, j=1, \ldots, n$.

Lemma 2.1 [11] Let $R_{i j}$ be the right ij-th cofactor of $\mathbf{A} \in \mathrm{M}(n, \mathbb{H})$, that is, $\operatorname{rdet}_{i} \mathbf{A}=\sum_{j=1}^{n} a_{i j} \cdot R_{i j}$ for all $i=1, \ldots, n$. Then

$$
R_{i j}=\left\{\begin{array}{cc}
-\operatorname{rdet}_{j} \mathbf{A}_{. j}^{i i}\left(\mathbf{a}_{. i}\right), & i \neq j, \\
\operatorname{rdet}_{k} \mathbf{A}^{i i}, & i=j,
\end{array}\right.
$$

where $\mathbf{A}_{. j}^{i i}\left(\mathbf{a}_{.}\right)$is obtained from $\mathbf{A}$ by replacing the $j$ th column with the $i$ th column, and then by deleting both the ith row and column, $k=\min \left\{I_{n} \backslash\{i\}\right\}$.

Lemma 2.2 [11] Let $L_{i j}$ be the left $i j$-th cofactor of $\mathbf{A} \in \mathrm{M}(n, \mathbb{H})$, that is, $\operatorname{cdet}_{j} \mathbf{A}=\sum_{i=1}^{n} L_{i j} \cdot a_{i j}$ for all $j=1, \ldots, n$. Then

$$
L_{i j}=\left\{\begin{array}{cc}
-\operatorname{cdet}_{i} \mathbf{A}_{i}^{j j}\left(\mathbf{a}_{j}\right), & i \neq j, \\
\operatorname{cdet}_{k} \mathbf{A}^{j j}, & i=j,
\end{array}\right.
$$

where $\mathbf{A}_{i .}^{j j}\left(\mathbf{a}_{j}.\right)$ is obtained from $\mathbf{A}$ by replacing the ith row with the $j$ th row, and then by deleting both the $j$ th row and column, $k=\min \left\{J_{n} \backslash\{j\}\right\}$. 
We recall some well-known definitions. The conjugate of a quaternion $a=$ $a_{0}+a_{1} i+a_{2} j+a_{3} k \in \mathbb{H}$ is defined by $\bar{a}=a_{o}-a_{1} i-a_{2} j-a_{3} k$. The Hermitian adjoint matrix of $\mathbf{A}=\left(a_{i j}\right) \in \mathbb{H}^{n \times m}$ is called the matrix $\mathbf{A}^{*}=\left(a_{i j}^{*}\right)_{m \times n}$ if $a_{i j}^{*}=\overline{a_{j i}}$ for all $i=1, \ldots, n$ and $j=1, \ldots, m$. The matrix $\mathbf{A}=\left(a_{i j}\right) \in \mathbb{H}^{n \times m}$ is Hermitian if $\mathbf{A}^{*}=\mathbf{A}$.

A following theorem has a key value in the theory of the column and row determinants.

Theorem 2.1 [11] If $\mathbf{A}=\left(a_{i j}\right) \in \mathrm{M}(n, \mathbb{H})$ is Hermitian, then $\operatorname{rdet}_{1} \mathbf{A}=$ $\cdots=\operatorname{rdet}_{n} \mathbf{A}=\operatorname{cdet}_{1} \mathbf{A}=\cdots=\operatorname{cdet}_{n} \mathbf{A} \in \mathbb{R}$.

Taking into account Theorem 2.1 we define the determinant of a Hermitian matrix by putting $\operatorname{det} \mathbf{A}:=\operatorname{rdet}_{i} \mathbf{A}=\operatorname{cdet}_{i} \mathbf{A}$ for all $i=1, \ldots, n$. This determinant of a Hermitian matrix coincides with the Moore determinant. The properties of the determinant of a Hermitian matrix are considered in [11] by means of the column and row determinants. Among them we note the following.

Theorem 2.2 [11] If the ith row of a Hermitian matrix $\mathbf{A} \in \mathrm{M}(n, \mathbb{H})$ is replaced with a left linear combination of its other rows, i.e. $\mathbf{a}_{i .}=c_{1} \mathbf{a}_{i_{1}}$. $\cdots+c_{k} \mathbf{a}_{i_{k}}$, where $c_{l} \in \mathbb{H}$ for all $l=1, \ldots, k$ and $\left\{i, i_{l}\right\} \subset I_{n}$, then

$\operatorname{cdet}_{i} \mathbf{A}_{i .}\left(c_{1} \cdot \mathbf{a}_{i_{1}}+\cdots+c_{k} \cdot \mathbf{a}_{i_{k}}.\right)=\operatorname{rdet}_{i} \mathbf{A}_{i .}\left(c_{1} \cdot \mathbf{a}_{i_{1}}+\cdots+c_{k} \cdot \mathbf{a}_{i_{k} .}\right)=$ 0 .

Theorem 2.3 [11] If the $j$ th column of a Hermitian matrix $\mathbf{A} \in \mathrm{M}(n, \mathbb{H})$ is replaced with a right linear combination of its other columns, i.e. $\mathbf{a}_{. j}=$ $\mathbf{a}_{. j_{1}} c_{1}+\cdots+\mathbf{a}_{\cdot j_{k}} c_{k}$, where $c_{l} \in \mathbb{H}$ for all $l=1, \ldots, k$ and $\left\{j, j_{l}\right\} \subset J_{n}$, then $\operatorname{cdet}_{i} \mathbf{A}_{. i}\left(\mathbf{a}_{i_{1}} \cdot c_{1}+\cdots+\mathbf{a}_{i_{k}} \cdot c_{k}\right)=\operatorname{rdet}_{i} \mathbf{A}_{. i}\left(\mathbf{a}_{i_{1}} \cdot c_{1}+\cdots+\mathbf{a}_{i_{k}} \cdot c_{k}\right)=$ 0 .

The following theorem about determinantal representation of an inverse matrix of Hermitian follows immediately from these properties.

Theorem 2.4 [11] If a Hermitian matrix $\mathbf{A} \in \mathrm{M}(n, \mathbb{H})$ is such that $\operatorname{det} \mathbf{A} \neq$ 0 , then there exist a unique right inverse matrix $(R \mathbf{A})^{-1}$ and a unique left inverse matrix $(L \mathbf{A})^{-1}$, and $(R \mathbf{A})^{-1}=(L \mathbf{A})^{-1}=: \mathbf{A}^{-1}$. They possess the following determinantal representations:

$$
(R \mathbf{A})^{-1}=\frac{1}{\operatorname{det} \mathbf{A}}\left(\begin{array}{cccc}
R_{11} & R_{21} & \cdots & R_{n 1} \\
R_{12} & R_{22} & \cdots & R_{n 2} \\
\cdots & \cdots & \cdots & \cdots \\
R_{1 n} & R_{2 n} & \cdots & R_{n n}
\end{array}\right),(L \mathbf{A})^{-1}=\frac{1}{\operatorname{det} \mathbf{A}}\left(\begin{array}{cccc}
L_{11} & L_{21} & \cdots & L_{n 1} \\
L_{12} & L_{22} & \cdots & L_{n 2} \\
\cdots & \cdots & \cdots & \cdots \\
L_{1 n} & L_{2 n} & \cdots & L_{n n}
\end{array}\right)
$$


Here $R_{i j}, L_{i j}$ are right and left $i j$-th cofactors of $\mathbf{A}$ respectively for all $i, j=$ $1, \ldots, n$.

To obtain determinantal representation of an arbitrary inverse matrix $\mathbf{A}^{-1}$, we consider the right $\mathbf{A} \mathbf{A}^{*}$ and left $\mathbf{A}^{*} \mathbf{A}$ corresponding Hermitian matrix.

Theorem 2.5 [11] If an arbitrary column of $\mathbf{A} \in \mathbb{H}^{m \times n}$ is a right linear combination of its other columns, or an arbitrary row of $\mathbf{A}^{*}$ is a left linear combination of its others, then $\operatorname{det} \mathbf{A}^{*} \mathbf{A}=0$.

Since the principal submatrices of a Hermitian matrix are Hermitian, the principal minor may be defined as the determinant of its principal submatrix by analogy to the commutative case. We introduce the rank by principal minors that is the maximal order of a nonzero principal minor of a Hermitian matrix. The following theorem determines a relationship between it and the rank of a matrix defining as ceiling amount of right-linearly independent columns or left-linearly independent rows which form basis.

Theorem 2.6 [11] A rank by principal minors of $\mathbf{A}^{*} \mathbf{A}$ is equal to its rank and a rank of $\mathbf{A} \in \mathbb{H}^{m \times n}$.

Theorem 2.7 [11] If $\mathbf{A} \in \mathbb{H}^{m \times n}$, then an arbitrary column of $\mathbf{A}$ is a right linear combination of its basis columns or an arbitrary row of $\mathbf{A}$ is a left linear combination of its basis rows.

The criterion of singularity of a Hermitian matrix is obtained.

Theorem 2.8 [11] The right-linearly independence of columns of $\mathbf{A} \in \mathbb{H}^{m \times n}$ or the left-linearly independence of rows of $\mathbf{A}^{*}$ is the necessary and sufficient condition for $\operatorname{det} \mathbf{A}^{*} \mathbf{A} \neq 0$.

Theorem 2.9 [11] If $\mathbf{A} \in \mathrm{M}(n, \mathbb{H})$, then $\operatorname{det} \mathbf{A} \mathbf{A}^{*}=\operatorname{det} \mathbf{A}^{*} \mathbf{A}$.

A concept of the double determinant is introduced by this theorem. This concept was initially introduced by L. Chen in [4].

Definition 2.3 The determinant of the corresponding Hermitian matrix of $\mathbf{A} \in \mathrm{M}(n, \mathbb{H})$ is called its double determinant, i.e. $\operatorname{ddet} \mathbf{A}:=\operatorname{det}\left(\mathbf{A}^{*} \mathbf{A}\right)=$ $\operatorname{det}\left(\mathbf{A A}^{*}\right)$. 
The relationship between the double determinant and the noncommutative determinants of E. Moore, E. Study and J. Diedonne is obtained, $\operatorname{ddet} \mathbf{A}=\operatorname{Mdet}\left(\mathbf{A}^{*} \mathbf{A}\right)=\operatorname{Sdet} \mathbf{A}=\operatorname{Ddet}^{2} \mathbf{A}$. But unlike those, the double determinant can be expanded along an arbitrary row or column by means of the column and row determinants.

Definition 2.4 Suppose $\mathbf{A} \in \mathrm{M}(n, \mathbb{H})$. We have a column expansion of $\operatorname{ddet} \mathbf{A}$ along the $j$ th column, $\operatorname{ddet} \mathbf{A}=\operatorname{cdet}_{j}\left(\mathbf{A}^{*} \mathbf{A}\right)=\sum_{i} \mathbb{L}_{i j} \cdot a_{i j}$, and a row expansion of it along the ith row, $\operatorname{ddet} \mathbf{A}=\operatorname{rdet}_{i}\left(\mathbf{A} \mathbf{A}^{*}\right)=\sum_{j} a_{i j} \cdot \mathbb{R}_{i j}$ for all $i, j=1, \ldots, n$. Then by definition of the left double ijth cofactor we put $\mathbb{L}_{i j}$ and by definition of the right double ijth cofactor we put $\mathbb{R}_{i j}$.

Theorem 2.10 [11] The necessary and sufficient condition of invertibility of $\mathbf{A} \in \mathrm{M}(n, \mathbb{H})$ is $\operatorname{ddet} \mathbf{A} \neq 0$. Then there exists $\mathbf{A}^{-1}=(L \mathbf{A})^{-1}=(R \mathbf{A})^{-1}$, where

$$
\begin{gathered}
(L \mathbf{A})^{-1}=\left(\mathbf{A}^{*} \mathbf{A}\right)^{-1} \mathbf{A}^{*}=\frac{1}{\operatorname{ddet} \mathbf{A}}\left(\begin{array}{cccc}
\mathbb{L}_{11} & \mathbb{L}_{21} & \ldots & \mathbb{L}_{n 1} \\
\mathbb{L}_{12} & \mathbb{L}_{22} & \ldots & \mathbb{L}_{n 2} \\
\ldots & \ldots & \ldots & \ldots \\
\mathbb{L}_{1 n} & \mathbb{L}_{2 n} & \ldots & \mathbb{L}_{n n}
\end{array}\right) \\
(R \mathbf{A})^{-1}=\mathbf{A}^{*}\left(\mathbf{A A}^{*}\right)^{-1}=\frac{1}{\operatorname{ddet} \mathbf{A}}\left(\begin{array}{cccc}
\mathbb{R}_{11} & \mathbb{R}_{21} & \ldots & \mathbb{R}_{n 1} \\
\mathbb{R}_{12} & \mathbb{R}_{22} & \ldots & \mathbb{R}_{n 2} \\
\ldots & \ldots & \ldots & \ldots \\
\mathbb{R}_{1 n} & \mathbb{R}_{2 n} & \ldots & \mathbb{R}_{n n}
\end{array}\right)
\end{gathered}
$$

and $\mathbb{L}_{i j}=\operatorname{cdet}_{j}\left(\mathbf{A}^{*} \mathbf{A}\right)_{. j}\left(\mathbf{a}_{. i}^{*}\right), \mathbb{R}_{i j}=\operatorname{rdet}_{i}\left(\mathbf{A} \mathbf{A}^{*}\right)_{i .}\left(\mathbf{a}_{j .}^{*}\right)$ for all $i, j=$ $1, \ldots, n$.

This theorem introduces the determinantal representations of an inverse matrix by the left (1) and right (2) double cofactors.

\section{The singular value decomposition and the Moore-Penrose inverse of a quaternion ma- trix}

Due to the noncommutativity of quaternions, there are two types of eigenvalues. 
Definition 3.1 Let $\mathbf{A} \in \mathrm{M}(n, \mathbb{H})$. A quaternion $\lambda$ is said to be a right eigenvalue of $\mathbf{A}$ if $\mathbf{A} \cdot \mathbf{x}=\mathbf{x} \cdot \lambda$ for some nonzero quaternion column-vector $\mathbf{x}$. Similarly $\lambda$ is a left eigenvalue if $\mathbf{A} \cdot \mathbf{x}=\lambda \cdot \mathbf{x}$.

The theory on the left eigenvalues of quaternion matrices has been investigated in particular in $9,15,18$. The theory on the right eigenvalues of quaternion matrices is more developed. In particular we note 2, 6, 21]. From this theory we cite the following propositions.

Proposition 3.1 [21] Let $\mathbf{A} \in \mathrm{M}(n, \mathbb{H})$ is Hermitian. Then $\mathbf{A}$ has exactly $n$ real right eigenvalues.

Definition 3.2 Suppose $\mathbf{U} \in \mathrm{M}(n, \mathbb{H})$. If $\mathbf{U}^{*} \mathbf{U}=\mathbf{U U}^{*}=\mathbf{I}$, then the matrix $\mathbf{U}$ is called unitary.

Proposition 3.2 [21] Let $\mathbf{A} \in \mathrm{M}(n, \mathbb{H})$ be given. Then, $\mathbf{A}$ is Hermitian if and only if there are a unitary matrix $\mathbf{U} \in \mathrm{M}(n, \mathbb{H})$ and a real diagonal matrix $\mathbf{D}=\operatorname{diag}\left(\lambda_{1}, \lambda_{2}, \ldots, \lambda_{n}\right)$ such that $\mathbf{A}=\mathbf{U D U}^{*}$, where $\lambda_{1}, \ldots, \lambda_{n}$ are right eigenvalues of $\mathbf{A}$.

Suppose $\mathbf{A} \in \mathrm{M}(n, \mathbb{H})$ is Hermitian and $\lambda \in \mathbb{R}$ is a right eigenvalue, then $\mathbf{A} \cdot \mathbf{x}=\mathbf{x} \cdot \lambda=\lambda \cdot \mathbf{x}$. This means that all right eigenvalues of a Hermitian matrix are its left eigenvalues as well. For real left eigenvalues, $\lambda \in \mathbb{R}$, the matrix $\lambda \mathbf{I}-\mathbf{A}$ is Hermitian.

Definition 3.3 If $t \in \mathbb{R}$, then for a Hermitian matrix $\mathbf{A}$ the polynomial $p_{\mathbf{A}}(t)=\operatorname{det}(t \mathbf{I}-\mathbf{A})$ is said to be the characteristic polynomial of $\mathbf{A}$.

The roots of the characteristic polynomial of a Hermitian matrix are its real left eigenvalues, which are its right eigenvalues as well. We shall investigate coefficients of the characteristic polynomial as in the commutative case (see, e.g. [13]). At first we prove the auxiliary lemma.

Lemma 3.1 Let $\mathbf{A} \in \mathrm{M}(n, \mathbb{H})$ be Hermitian and the columns $i_{1}, \ldots, i_{k}$ of $\mathbf{A}$ coincide with the unit vectors $\mathbf{e}_{i_{1}}, \ldots, \mathbf{e}_{i_{k}}$. Then $\operatorname{det} \mathbf{A}$ equals a principal minor obtained from $\mathbf{A}$ by deleting the rows and columns $i_{1}, \ldots, i_{k}$.

Proof. We claim that if $\mathbf{A} \in \mathrm{M}(n, \mathbb{H})$ is Hermitian and the columns $i_{1}, \ldots, i_{k}$ of $\mathbf{A}$ coincide with the unit column vectors $\mathbf{e}_{i_{1}}, \ldots, \mathbf{e}_{i_{k}}$ respectively, then the rows $i_{1}, \ldots, i_{k}$ coincide with the unit row vectors $\mathbf{e}_{i_{1}}, \ldots, \mathbf{e}_{i_{k}}$ as well. Using 
Lemma 2.1 we expand $\operatorname{det} \mathbf{A}$ along the $i_{1}$ th column, where $a_{i_{1} k}=0$ for all $k \neq i_{1}$ and $a_{i_{1} i_{1}}=1$. Then we obtain

$$
\begin{gathered}
\operatorname{det} \mathbf{A}=\operatorname{cdet}_{i_{1}} \mathbf{A}= \\
=-\operatorname{cdet}_{i_{1}} \mathbf{A}_{i_{1}}^{11}\left(\mathbf{a}_{1 .}\right) \cdot 0+\cdots+\operatorname{cotet}_{1} \mathbf{A}^{i_{1} i_{1}} \cdot 1+\cdots-\cot _{i_{1}} \mathbf{A}_{i_{1} .}^{n n}\left(\mathbf{a}_{n} .\right) \cdot 0= \\
=\operatorname{cdet}_{1} \mathbf{A}^{i_{1} i_{1}} .
\end{gathered}
$$

Since the submatrix $\mathbf{A}^{i_{1} i_{1}}$ is obtained from $\mathbf{A}$ by deleting both the $i_{1}$-th rows and columns, by Theorem 2.1 it follows that $\operatorname{cotet}_{1} \mathbf{A}^{i_{1} i_{1}}=\operatorname{det} \mathbf{A}^{i_{1} i_{1}}$. Now we calculate this principal minor expanding along the $i_{2}$-th column. Similarly to above we have that $\operatorname{det} \mathbf{A}$ is equal to a principal minor obtained from $\mathbf{A}$ by deleting both the $i_{1}$ th and $i_{2}$ th rows and columns. Continuing this line of reasoning we complete the proof of the lemma.

Taking into account Lemma 3.1 we can prove the following theorem by analogy to the commutative case (see, e.g. [13]).

Theorem 3.1 If $\mathbf{A} \in \mathrm{M}(n, \mathbb{H})$ is Hermitian, then $p_{\mathbf{A}}(t)=t^{n}-d_{1} t^{n-1}+$ $d_{2} t^{n-2}-\cdots+(-1)^{n} d_{n}$, where $d_{r}$ is the sum of principle minors of $\mathbf{A}$ of order $r, 1 \leq r<n$, and $d_{n}=\operatorname{det} \mathbf{A}$.

For any quaternion matrix $\mathbf{A} \in \mathrm{M}(n, \mathbb{H})$, the eigenvalues of $\mathbf{A}^{*} \mathbf{A}$ are all nonnegative real numbers [17].

Definition 3.4 Let $\mathbf{A} \in \mathbb{H}^{m \times n}$. The nonnegative square roots of the $n$ eigenvalues of $\mathbf{A}^{*} \mathbf{A}$ are called the singular values of $\mathbf{A}$.

A key value for a determinantal representation of the Moore-Penrose inverse over the quaternion skew field is the following singular value decomposition (SVD) theorem.

Theorem 3.2 [17, 21] (SVD) Let $\mathbf{A} \in \mathbb{H}_{r}^{m \times n}$. Then there exist unitary quaternion matrices $\mathbf{U}_{1} \in \mathbb{H}^{m \times m}$ and $\mathbf{U}_{2} \in \mathbb{H}^{n \times n}$ such that

$$
\mathbf{U}_{1} \mathbf{A U}_{2}=\left[\begin{array}{ll}
\mathbf{D}_{r} & \mathbf{0} \\
\mathbf{0} & \mathbf{0}
\end{array}\right] \in \mathbb{H}^{m \times n}
$$

where $\mathbf{D}_{r}=\operatorname{diag}\left(\sigma_{1}, \sigma_{2}, \ldots, \sigma_{r}\right), \sigma_{1} \geq \sigma_{2} \geq \cdots \geq \sigma_{r}>0$, and $\sigma_{1}, \sigma_{2}, \ldots, \sigma_{r}$ are the all nonzero singular values of $\mathbf{A}$. 
As unitary matrices are invertible, the equality (3) can be written as follows

$$
\mathbf{A}=\mathbf{V} \mathbf{\Sigma} \mathbf{W}^{*}
$$

where $\mathbf{V} \in \mathbb{H}^{m \times m}$ and $\mathbf{W} \in \mathbb{H}^{n \times n}$ are unitary matrices, and the matrix $\boldsymbol{\Sigma}=$ $\left(\sigma_{i j}\right) \in \mathbb{H}_{r}^{m \times n}$ is such that $\sigma_{11} \geq \sigma_{22} \geq \cdots \geq \sigma_{r r}>\sigma_{r+1 r+1}=\cdots=\sigma_{q q}=0$, $q=\min \{n, m\}$.

We get the following lemmas, which have the analogues in the complex case [3].

Lemma 3.2 Suppose $\mathbf{A} \in \mathbb{H}^{m \times n}$ has the singular value decomposition, $\mathbf{A}=$ $\mathbf{V} \boldsymbol{\Sigma} \mathbf{W}^{*}$. Let $\mathbf{A}^{+}=\mathbf{W} \cdot \boldsymbol{\Sigma}^{+} \cdot \mathbf{V}^{*}$, where $\boldsymbol{\Sigma}^{+} \in \mathbb{H}^{n \times m}$ is obtained from $\boldsymbol{\Sigma}$ by transposition and replacing positive entries of $\mathbf{\Sigma}$ by reciprocal. Then for $\mathbf{A}^{+}$ the following conditions are true

1) $\left(\mathbf{A A}^{+}\right)^{*}=\mathbf{A} \mathbf{A}^{+}$

2) $\left(\mathbf{A}^{+} \mathbf{A}\right)^{*}=\mathbf{A}^{+} \mathbf{A}$

3) $\mathbf{A A}^{+} \mathbf{A}=\mathbf{A}$

4) $\mathbf{A}^{+} \mathbf{A} \mathbf{A}^{+}=\mathbf{A}^{+}$.

Proof. We obviously have $\left(\Sigma^{T}\right)^{*}=\Sigma$ and $\left(\left(\Sigma^{+}\right)^{T}\right)^{*}=\Sigma^{+}$for $\boldsymbol{\Sigma}$ from the SVD by (4) and $\boldsymbol{\Sigma}^{+}$. Then it follows that

$$
\begin{aligned}
\left(\mathbf{A} A^{+}\right)^{*} & =\left(\mathbf{V} \boldsymbol{\Sigma} \mathbf{W}^{*} \mathbf{W} \boldsymbol{\Sigma}^{+} \mathbf{V}^{*}\right)^{*}=\left(\mathbf{V} \boldsymbol{\Sigma} \mathbf{I} \boldsymbol{\Sigma}^{+} \mathbf{V}^{*}\right)^{*}=\left(\mathbf{V}\left(\boldsymbol{\Sigma}^{+}\right)^{T} \boldsymbol{\Sigma}^{T} \mathbf{V}^{*}\right)^{*}= \\
& =\left(\mathbf{V}\left(\boldsymbol{\Sigma}^{+}\right)^{T} \mathbf{W}^{*} \mathbf{W} \boldsymbol{\Sigma}^{T} \mathbf{V}^{*}\right)^{*}=\mathbf{V} \boldsymbol{\Sigma} \mathbf{W}^{*} \mathbf{W} \boldsymbol{\Sigma}^{+} \mathbf{V}^{*}=\mathbf{A} \mathbf{A}^{+}
\end{aligned}
$$

The proof of 1) is completed. By analogy we can prove 2).

Now we prove the condition 3). Note that $\boldsymbol{\Sigma} \boldsymbol{\Sigma}^{+}=\left[\begin{array}{cc}\mathbf{I}_{r} & \mathbf{0} \\ \mathbf{0} & \mathbf{0}\end{array}\right] \in \mathbb{H}^{m \times m}$. This implies $\boldsymbol{\Sigma} \boldsymbol{\Sigma}^{+} \boldsymbol{\Sigma}=\boldsymbol{\Sigma}$, then $\mathbf{A A}^{+} \mathbf{A}=\mathbf{V} \boldsymbol{\Sigma} \mathbf{W}^{*} \cdot \mathbf{W} \boldsymbol{\Sigma}^{+} \mathbf{V}^{*} \cdot \mathbf{V} \boldsymbol{\Sigma} \mathbf{W}^{*}=$ $\mathbf{V} \cdot \boldsymbol{\Sigma} \boldsymbol{\Sigma}^{+} \boldsymbol{\Sigma} \cdot \mathbf{W}^{*}=\mathbf{V} \cdot \boldsymbol{\Sigma} \cdot \mathbf{W}^{*}=\mathbf{A}$.

By analogy to 3) can be prove the condition 4)

Lemma 3.3 There exists a unique matrix $\mathbf{A}^{+}$that satisfies conditions 1)-4) in (5). 
Proof. Suppose that both matrices $\mathbf{B} \in \mathbb{H}^{n \times m}$ and $\mathbf{C} \in \mathbb{H}^{n \times m}$ satisfy conditions 1)-4) in Lemma 3.2. Then we have

$$
\begin{aligned}
& \mathbf{B}=\mathbf{B A B}=\mathbf{B}(\mathbf{A B})^{*}=\mathbf{B B}^{*} \mathbf{A}^{*}=\mathbf{B B}^{*}(\mathbf{A C A})^{*}=\mathbf{B B}^{*} \mathbf{A}^{*} \mathbf{C}^{*} \mathbf{A}^{*}= \\
= & \mathbf{B}(\mathbf{A B})^{*}(\mathbf{A C})^{*}=\mathbf{B A B A C}=\mathbf{B A C}=\mathbf{B A C A C}=(\mathbf{B A})^{*}(\mathbf{C A})^{*} \mathbf{C}= \\
= & \mathbf{A}^{*} \mathbf{B}^{*} \mathbf{A}^{*} \mathbf{C}^{*} \mathbf{C}=(\mathbf{A B A})^{*} \mathbf{C}^{*} \mathbf{C}=\mathbf{A}^{*} \mathbf{C}^{*} \mathbf{C}=(\mathbf{C A})^{*} \mathbf{C}=\mathbf{C A C}=\mathbf{C} .
\end{aligned}
$$

Definition 3.5 Let $\mathbf{A} \in \mathbb{H}^{m \times n}$. The matrix $\mathbf{A}^{+}$is called the Moore-Penrose inverse if it satisfies all conditions in (5).

By analogy to the complex case [3] we have the theorem about the limit representation of the Moore-Penrose inverse.

Theorem 3.3 If $\mathbf{A} \in \mathbb{H}^{m \times n}$ and $\mathbf{A}^{+}$is its Moore-Penrose inverse, then $\mathbf{A}^{+}=\lim _{\alpha \rightarrow 0} \mathbf{A}^{*}\left(\mathbf{A} \mathbf{A}^{*}+\alpha \mathbf{I}\right)^{-1}=\lim _{\alpha \rightarrow 0}\left(\mathbf{A}^{*} \mathbf{A}+\alpha \mathbf{I}\right)^{-1} \mathbf{A}^{*}$, where $\alpha \in \mathbb{R}_{+}$.

Proof. Suppose $\mathbf{A}=\mathbf{V} \boldsymbol{\Sigma} \mathbf{W}^{*}$, then $\mathbf{A}^{*}=\mathbf{W} \boldsymbol{\Sigma}^{*} \mathbf{V}^{*}$ and $\mathbf{A}^{+}=\mathbf{W} \boldsymbol{\Sigma}^{+} \mathbf{V}^{*}$. Since $\mathbf{V}$ is unitary, then $\mathbf{V}^{*}=\mathbf{V}^{-1}$. We have

$$
\begin{aligned}
& \mathbf{A}^{*}\left(\mathbf{A} \mathbf{A}^{*}+\alpha \mathbf{I}\right)^{-1}=\mathbf{W} \boldsymbol{\Sigma} \mathbf{V}^{*} \cdot\left(\mathbf{V} \boldsymbol{\Sigma} \cdot \mathbf{W}^{*} \mathbf{W} \cdot \boldsymbol{\Sigma} \mathbf{V}^{*}+\alpha \mathbf{I}\right)^{-1}= \\
& =\mathbf{W} \boldsymbol{\Sigma} \mathbf{V}^{*} \cdot\left(\mathbf{V}\left(\boldsymbol{\Sigma} \boldsymbol{\Sigma}^{*}+\alpha \mathbf{I}\right) \mathbf{V}^{*}\right)^{-1}=\mathbf{W} \boldsymbol{\Sigma}\left(\boldsymbol{\Sigma} \boldsymbol{\Sigma}^{*}+\alpha \mathbf{I}\right)^{-1} \mathbf{V}^{*}
\end{aligned}
$$

Consider the matrix

$$
\boldsymbol{\Sigma}\left(\boldsymbol{\Sigma} \boldsymbol{\Sigma}^{*}+\alpha \mathbf{I}\right)^{-1}=\left(\begin{array}{lllll}
\frac{\lambda_{1}}{\lambda_{1}^{2}+\alpha} & \ldots & 0 & & \\
\cdots & \ldots & \ldots & & \mathbf{0} \\
0 & \ldots & \frac{\lambda_{r}}{\lambda_{r}^{2}+\alpha} & & \vdots \\
& \vdots & & \ddots & \\
& \mathbf{0} & & & \mathbf{0}
\end{array}\right) .
$$

It is obviously that $\lim _{\alpha \rightarrow 0} \boldsymbol{\Sigma}\left(\boldsymbol{\Sigma} \boldsymbol{\Sigma}^{*}+\alpha \mathbf{I}\right)^{-1}=\boldsymbol{\Sigma}^{+}$. This implies $\lim _{\alpha \rightarrow 0} \mathbf{A}^{*}\left(\mathbf{A} \mathbf{A}^{*}+\alpha \mathbf{I}\right)^{-1}=$ $\lim _{\alpha \rightarrow 0} \mathbf{W} \boldsymbol{\Sigma}\left(\boldsymbol{\Sigma} \boldsymbol{\Sigma}^{*}+\alpha \mathbf{I}\right)^{-1} \mathbf{V}^{*}=\mathbf{A}^{+}$.

By analogy we can prove that $\mathbf{A}^{+}=\lim _{\alpha \rightarrow 0}\left(\mathbf{A}^{*} \mathbf{A}+\alpha \mathbf{I}\right)^{-1} \mathbf{A}^{*}$

Corollary 3.1 If $\mathbf{A} \in \mathbb{H}^{m \times n}$, then the following statements are true.

i) If $\operatorname{rank} \mathbf{A}=\mathrm{n}$, then $\mathbf{A}^{+}=\left(\mathbf{A}^{*} \mathbf{A}\right)^{-1} \mathbf{A}^{*}$.

ii) If $\operatorname{rank} \mathbf{A}=\mathrm{m}$, then $\mathbf{A}^{+}=\mathbf{A}^{*}\left(\mathbf{A} \mathbf{A}^{*}\right)^{-1}$.

iii) If $\operatorname{rank} \mathbf{A}=\mathrm{n}=\mathrm{m}$, then $\mathbf{A}^{+}=\mathbf{A}^{-1}$. 


\section{Determinantal representation of the Moore- Penrose inverse.}

Lemma 4.1 If $\mathbf{A} \in \mathbb{H}_{r}^{m \times n}$, then $\operatorname{rank}\left(\mathbf{A}^{*} \mathbf{A}\right)_{. i}\left(\mathbf{a}_{. j}^{*}\right) \leq r$.

Proof. Let's lead elementary transformations of the matrix $\left(\mathbf{A}^{*} \mathbf{A}\right)_{. i}\left(\mathbf{a}_{. j}^{*}\right)$ right-multiplying it by elementary unimodular matrices $\mathbf{P}_{i k}\left(-a_{j k}\right), k \neq j$. The matrix $\mathbf{P}_{i k}\left(-a_{j k}\right)$ has $-a_{j k}$ in the $(i, k)$ entry, 1 in all diagonal entries, and 0 in others. It is the matrix of an elementary transformation. Rightmultiplying a matrix by $\mathbf{P}_{i k}\left(-a_{j k}\right)$, where $k \neq j$, means adding to $k$-th column its $i$-th column right-multiplying on $-a_{j k}$. Then we get

$$
\left(\mathbf{A}^{*} \mathbf{A}\right)_{. i}\left(\mathbf{a}_{. j}^{*}\right) \cdot \prod_{k \neq i} \mathbf{P}_{i k}\left(-a_{j k}\right)=\left(\begin{array}{lllll}
\sum_{k \neq j} a_{1 k}^{*} a_{k 1} & \ldots & a_{1 j}^{*} & \ldots & \sum_{k \neq j} a_{1 k}^{*} a_{k n} \\
\ldots & \ldots & \ldots & \ldots & \ldots \\
\sum_{k \neq j} a_{n k}^{*} a_{k 1} & \ldots & a_{n j}^{*} & \ldots & \sum_{k \neq j} a_{n k}^{*} a_{k n}
\end{array}\right) .
$$

The obtained matrix has the following factorization.

$$
\begin{gathered}
\left(\begin{array}{llllll}
\sum_{k \neq j} a_{1 k}^{*} a_{k 1} & \ldots & a_{1 j}^{*} & \ldots & \sum_{k \neq j} a_{1 k}^{*} a_{k n} \\
\ldots & & \ldots & \ldots & \ldots & \ldots \\
\sum_{k \neq j} a_{n k}^{*} a_{k 1} & \ldots & a_{n j}^{*} & \ldots & \sum_{k \neq j} a_{n k}^{*} a_{k n}
\end{array}\right)= \\
=\left(\begin{array}{lllll}
a_{11}^{*} & a_{12}^{*} & \ldots & a_{1 m}^{*} \\
a_{21}^{*} & a_{22}^{*} & \ldots & a_{2 m}^{*} \\
\ldots & \ldots & \ldots & \ldots \\
a_{n 1}^{*} & a_{n 2}^{*} & \ldots & a_{n m}^{*}
\end{array}\right)\left(\begin{array}{lllll}
a_{11} & \ldots & 0 & \ldots & a_{n 1} \\
\ldots & \ldots & \ldots & \ldots & \ldots \\
0 & \ldots & 1 & \ldots & 0 \\
\ldots & \ldots & \ldots & \ldots & \ldots \\
a_{m 1} & \ldots & 0 & \ldots & a_{m n}
\end{array}\right) j-t h . \\
\text { Denote by } \tilde{\mathbf{A}}:=\left(\begin{array}{lllll}
a_{11} & \ldots & 0 & \ldots & a_{n 1} \\
\ldots & \ldots & \ldots & \ldots & \ldots \\
0 & \ldots & 1 & \ldots & 0 \\
\ldots & \ldots & \ldots & \ldots & \ldots \\
a_{m 1} & \ldots & 0 & \ldots & a_{m n}
\end{array}\right) j-t h . \\
i-t h
\end{gathered}
$$

tained from $\mathbf{A}$ by replacing all entries of the $j$ th row and of the $i$ th column 
with zeroes except that the $(j, i)$ entry equals 1 . Elementary transformations of a matrix do not change its rank and the rank of a matrix product does not exceed a rank of each factor. It follows that $\operatorname{rank}\left(\mathbf{A}^{*} \mathbf{A}\right)_{. i}\left(\mathbf{a}_{. j}^{*}\right) \leq$ $\min \left\{\operatorname{rank} \mathbf{A}^{*}, \operatorname{rank} \tilde{\mathbf{A}}\right\}$. It is obvious that $\operatorname{rank} \tilde{\mathbf{A}} \geq \operatorname{rank} \mathbf{A}=\operatorname{rank} \mathbf{A}^{*}$. Taking into account Theorem 2.6 we obtain $\operatorname{rank} \mathbf{A}^{*} \mathbf{A}=\operatorname{rank} \mathbf{A}$. This completes the proof.

The following lemma is proved in the same way.

Lemma 4.2 If $\mathbf{A} \in \mathbb{H}_{r}^{m \times n}$, then $\operatorname{rank}\left(\mathbf{A A}^{*}\right)_{. i}\left(\mathbf{a}_{. j}^{*}\right) \leq r$.

We shall use the following notations. Let $\alpha:=\left\{\alpha_{1}, \ldots, \alpha_{k}\right\} \subseteq\{1, \ldots, m\}$ and $\beta:=\left\{\beta_{1}, \ldots, \beta_{k}\right\} \subseteq\{1, \ldots, n\}$ be subsets of the order $1 \leq k \leq \min \{m, n\}$. By $\mathbf{A}_{\beta}^{\alpha}$ denote the submatrix of $\mathbf{A}$ determined by the rows indexed by $\alpha$ and the columns indexed by $\beta$. Then $\mathbf{A}_{\alpha}^{\alpha}$ denotes the principal submatrix determined by the rows and columns indexed by $\alpha$. If $\mathbf{A} \in \mathrm{M}(n, \mathbb{H})$ is Hermitian, then by $\left|\mathbf{A}_{\alpha}^{\alpha}\right|$ denote the corresponding principal minor of $\operatorname{det} \mathbf{A}$. For $1 \leq k \leq$ $n$, denote by $L_{k, n}:=\left\{\alpha: \alpha=\left(\alpha_{1}, \ldots, \alpha_{k}\right), 1 \leq \alpha_{1} \leq \cdots \leq \alpha_{k} \leq n\right\}$, the collection of strictly increasing sequences of $k$ integers chosen from $\{1, \ldots, n\}$. For fixed $i \in \alpha$ and $j \in \beta$, let $I_{r, m}\{i\}:=\left\{\alpha: \alpha \in L_{r, m}, i \in \alpha\right\}, \quad J_{r, n}\{j\}:=$ $\left\{\beta: \beta \in L_{r, n}, j \in \beta\right\}$.

Lemma 4.3 If $\mathbf{A} \in \mathbb{H}^{m \times n}$ and $t \in \mathbb{R}$, then

$$
\operatorname{cdet}_{i}\left(t \mathbf{I}+\mathbf{A}^{*} \mathbf{A}\right)_{. i}\left(\mathbf{a}_{. j}^{*}\right)=c_{1}^{(i j)} t^{n-1}+c_{2}^{(i j)} t^{n-2}+\cdots+c_{n}^{(i j)},
$$

where $c_{n}^{(i j)}=\operatorname{cdet}_{i}\left(\mathbf{A}^{*} \mathbf{A}\right)_{. i}\left(\mathbf{a}_{\cdot j}^{*}\right)$ and $c_{k}^{(i j)}=\sum_{\beta \in J_{k, n}\{i\}} \operatorname{cdet}_{i}\left(\left(\mathbf{A}^{*} \mathbf{A}\right)_{. i}\left(\mathbf{a}_{\cdot j}^{*}\right)\right)_{\beta}^{\beta}$ for all $k=1, \ldots, n-1, i=1, \ldots, n$, and $j=1, \ldots, m$.

Proof. Denote by $\mathbf{b}_{. i}$ the $i$-th column of the Hermitian matrix $\mathbf{A}^{*} \mathbf{A}=$ : $\left(b_{i j}\right)_{n \times n}$. Consider the Hermitian matrix $\left(t \mathbf{I}+\mathbf{A}^{*} \mathbf{A}\right)_{. i}\left(\mathbf{b}_{. i}\right) \in \mathbb{H}^{n \times n}$. It differs from $\left(t \mathbf{I}+\mathbf{A}^{*} \mathbf{A}\right)$ by an entry $b_{i i}$. Taking into account Theorem 3.1 we obtain

$$
\operatorname{det}\left(t \mathbf{I}+\mathbf{A}^{*} \mathbf{A}\right)_{. i}\left(\mathbf{b}_{. i}\right)=d_{1} t^{n-1}+d_{2} t^{n-2}+\cdots+d_{n},
$$

where $d_{k}=\sum_{\beta \in J_{k, n}\{i\}} \operatorname{det}\left(\mathbf{A}^{*} \mathbf{A}\right)_{\beta}^{\beta}$ is the sum of all principal minors of order $k$ that contain the $i$-th column for all $k=1, \ldots, n-1$ and $d_{n}=\operatorname{det}\left(\mathbf{A}^{*} \mathbf{A}\right)$. 
Consequently we have $\mathbf{b}_{. i}=\left(\begin{array}{l}\sum_{l} a_{1 l}^{*} a_{l i} \\ \sum_{l} a_{2 l}^{*} a_{l i} \\ \vdots \\ \sum_{l} a_{n l}^{*} a_{l i}\end{array}\right)=\sum_{l} \mathbf{a}_{. l}^{*} a_{l i}$, where $\mathbf{a}_{. l}^{*}$ is the $l$ th column-vector of $\mathbf{A}^{*}$ for all $l=1, \ldots, m$. Taking into account Theorem 2.1, Lemma 2.1 and Proposition 2.2 we obtain on the one hand

$$
\begin{gathered}
\operatorname{det}\left(t \mathbf{I}+\mathbf{A}^{*} \mathbf{A}\right)_{. i}\left(\mathbf{b}_{. i}\right)=\operatorname{cdet}_{i}\left(t \mathbf{I}+\mathbf{A}^{*} \mathbf{A}\right)_{. i}\left(\mathbf{b}_{. i}\right)= \\
=\sum_{l} \operatorname{cdet}_{i}\left(t \mathbf{I}+\mathbf{A}^{*} \mathbf{A}\right)_{. l}\left(\mathbf{a}_{. l}^{*} a_{l i}\right)=\sum_{l} \operatorname{cdet}_{i}\left(t \mathbf{I}+\mathbf{A}^{*} \mathbf{A}\right)_{. i}\left(\mathbf{a}_{. l}^{*}\right) \cdot a_{l i}
\end{gathered}
$$

On the other hand having changed the order of summation, we get for all $k=1, \ldots, n-1$

$$
\begin{gathered}
d_{k}=\sum_{\beta \in J_{k, n}\{i\}} \operatorname{det}\left(\mathbf{A}^{*} \mathbf{A}\right){ }_{\beta}^{\beta}=\sum_{\beta \in J_{k, n}\{i\}} \operatorname{cdet}_{i}\left(\mathbf{A}^{*} \mathbf{A}\right)_{\beta}^{\beta}= \\
\sum_{\beta \in J_{k, n}\{i\}} \sum_{l} \operatorname{cdet}_{i}\left(\left(\mathbf{A}^{*} \mathbf{A}\right)_{. i}\left(\mathbf{a}_{. l}^{*} a_{l i}\right)\right)_{\beta}^{\beta}=\sum_{l} \sum_{\beta \in J_{k, n}\{i\}} \operatorname{cdet}_{i}\left(\left(\mathbf{A}^{*} \mathbf{A}\right)_{. i}\left(\mathbf{a}_{. l}^{*}\right)\right)_{\beta}^{\beta} \cdot a_{l i} .
\end{gathered}
$$

By substituting (8) and (9) in (7), and equating factors at $a_{l i}$ when $l=j$, we obtain the equality (6).

By analogy the following lemma can be proved.

Lemma 4.4 If $\mathbf{A} \in \mathbb{H}^{m \times n}$ and $t \in \mathbb{R}$, then

$$
\operatorname{rdet}_{j}\left(t \mathbf{I}+\mathbf{A A}^{*}\right)_{j .}\left(\mathbf{a}_{i .}^{*}\right)=r_{1}^{(i j)} t^{n-1}+r_{2}^{(i j)} t^{n-2}+\cdots+r_{n}^{(i j)},
$$

where $r_{n}^{(i j)}=\operatorname{rdet}_{j}\left(\mathbf{A} \mathbf{A}^{*}\right)_{j} .\left(\mathbf{a}_{i .}^{*}\right)$ and $r_{k}^{(i j)}=\sum_{\alpha \in I_{r, m}\{j\}} \operatorname{rdet}_{j}\left(\left(\mathbf{A} \mathbf{A}^{*}\right)_{j} .\left(\mathbf{a}_{i .}^{*}\right)\right){ }_{\alpha}^{\alpha}$ for all $k=1, \ldots, n-1, i=1, \ldots, n$, and $j=1, \ldots, m$.

Theorem 4.1 If $\mathbf{A} \in \mathbb{H}_{r}^{m \times n}$, then the Moore-Penrose inverse $\mathbf{A}^{+}=\left(a_{i j}^{+}\right) \in$ $\mathbb{H}^{n \times m}$ possess the following determinantal representations:

$$
a_{i j}^{+}=\frac{\sum_{\beta \in J_{r, n}\{i\}} \operatorname{cdet}_{i}\left(\left(\mathbf{A}^{*} \mathbf{A}\right)_{. i}\left(\mathbf{a}_{. j}^{*}\right)\right)_{\beta}^{\beta}}{\sum_{\beta \in J_{r, n}}\left|\left(\mathbf{A}^{*} \mathbf{A}\right)_{\beta}^{\beta}\right|},
$$


or

$$
a_{i j}^{+}=\frac{\sum_{\alpha \in I_{r, m}\{j\}} \operatorname{rdet}_{j}\left(\left(\mathbf{A} \mathbf{A}^{*}\right)_{j .}\left(\mathbf{a}_{i .}^{*}\right)\right)_{\alpha}^{\alpha}}{\sum_{\alpha \in I_{r, m}}\left|\left(\mathbf{A} \mathbf{A}^{*}\right)_{\alpha}^{\alpha}\right|} .
$$

Proof. At first we prove (10). Using Theorem 3.3, we get $\mathbf{A}^{+}=\lim _{\alpha \rightarrow 0}\left(\alpha \mathbf{I}+\mathbf{A}^{*} \mathbf{A}\right)^{-1} \mathbf{A}^{*}$. The matrix $\left(\alpha \mathbf{I}+\mathbf{A}^{*} \mathbf{A}\right) \in \mathbb{H}^{n \times n}$ is a full-rank Hermitian matrix. Taking into account Theorem 2.4 it has an inverse, which we represent as a left inverse matrix

$$
\left(\alpha \mathbf{I}+\mathbf{A}^{*} \mathbf{A}\right)^{-1}=\frac{1}{\operatorname{det}\left(\alpha \mathbf{I}+\mathbf{A}^{*} \mathbf{A}\right)}\left(\begin{array}{llll}
L_{11} & L_{21} & \ldots & L_{n 1} \\
L_{12} & L_{22} & \ldots & L_{n 2} \\
\ldots & \ldots & \ldots & \ldots \\
L_{1 n} & L_{2 n} & \ldots & L_{n n}
\end{array}\right),
$$

where $L_{i j}$ is a left $i j$ th cofactor of a matrix $\alpha \mathbf{I}+\mathbf{A}^{*} \mathbf{A}$. Then we have

$$
\begin{aligned}
& \left(\alpha \mathbf{I}+\mathbf{A}^{*} \mathbf{A}\right)^{-1} \mathbf{A}^{*}= \\
& \left(\begin{array}{lllll}
\sum_{k=1}^{n} L_{k 1} a_{k 1}^{*} & \sum_{k=1}^{n} L_{k 1} a_{k 2}^{*} & \ldots & \sum_{k=1}^{n} L_{k 1} a_{k m}^{*} \\
\sum_{k=1}^{n} L_{k 2} a_{k 1}^{*} & \sum_{k=1}^{n} L_{k 2} a_{k 2}^{*} & \ldots & \sum_{k=1}^{n} L_{k 2} a_{k m}^{*} \\
\ldots & \ldots & \ldots & \ldots \\
\sum_{k=1}^{n} L_{k n} a_{k 1}^{*} & \sum_{k=1}^{n} L_{k n} a_{k 2}^{*} & \ldots & \sum_{k=1}^{n} L_{k n} a_{k m}^{*}
\end{array}\right) .
\end{aligned}
$$

Using the definition of a left cofactor, we obtain

$$
\mathbf{A}^{+}=\lim _{\alpha \rightarrow 0}\left(\begin{array}{lll}
\frac{\operatorname{cdet}_{1}\left(\alpha \mathbf{I}+\mathbf{A}^{*} \mathbf{A}\right)_{.1}\left(\mathbf{a}_{.1}^{*}\right)}{\operatorname{det}\left(\alpha \mathbf{I}+\mathbf{A}^{*} \mathbf{A}\right)} & \ldots & \frac{\operatorname{cdet}_{1}\left(\alpha \mathbf{I}+\mathbf{A}^{*} \mathbf{A}\right)_{.1}\left(\mathbf{a}_{\cdot m}^{*}\right)}{\operatorname{det}\left(\alpha \mathbf{I}+\mathbf{A}^{*} \mathbf{A}\right)} \\
\ldots & \ldots & \ldots \\
\frac{\operatorname{cdet}_{n}\left(\alpha \mathbf{I}+\mathbf{A}^{*} \mathbf{A}\right)_{. n}\left(\mathbf{a}_{\cdot .1}^{*}\right)}{\operatorname{det}\left(\alpha \mathbf{I}+\mathbf{A}^{*} \mathbf{A}\right)} & \ldots & \frac{\operatorname{cdet}_{n}\left(\alpha \mathbf{I}+\mathbf{A}^{*} \mathbf{A}\right)_{. n}\left(\mathbf{a}_{\cdot m}^{*}\right)}{\operatorname{det}\left(\alpha \mathbf{I}+\mathbf{A}^{*} \mathbf{A}\right)}
\end{array}\right) .
$$

By Theorem 3.1 we have $\operatorname{det}\left(\alpha \mathbf{I}+\mathbf{A}^{*} \mathbf{A}\right)=\alpha^{n}+d_{1} \alpha^{n-1}+d_{2} \alpha^{n-2}+\cdots+d_{n}$, where $d_{k}=\sum_{\beta \in J_{k, n}}\left|\left(\mathbf{A}^{*} \mathbf{A}\right)_{\beta}^{\beta}\right|$ is a sum of principal minors of $\mathbf{A}^{*} \mathbf{A}$ of order $k$ for all $k=1, \ldots, n-1$ and $d_{n}=\operatorname{det} \mathbf{A}^{*} \mathbf{A}$. Since $\operatorname{rank} \mathbf{A}^{*} \mathbf{A}=\operatorname{rank} \mathbf{A}=r$ and $d_{n}=d_{n-1}=\cdots=d_{r+1}=0$, it follows that $\operatorname{det}\left(\alpha \mathbf{I}+\mathbf{A}^{*} \mathbf{A}\right)=\alpha^{n}+$ $d_{1} \alpha^{n-1}+d_{2} \alpha^{n-2}+\cdots+d_{r} \alpha^{n-r}$. Using (6) we get $\operatorname{cdet}_{i}\left(\alpha \mathbf{I}+\mathbf{A}^{*} \mathbf{A}\right)_{. i}\left(\mathbf{a}_{. j}^{*}\right)=$ 
$c_{1}^{(i j)} \alpha^{n-1}+c_{2}^{(i j)} \alpha^{n-2}+\cdots+c_{n}^{(i j)}$ for all $i=1, \ldots, n$ and $j=1, \ldots, m$, where $c_{k}^{(i j)}=\sum_{\beta \in J_{k, n}\{i\}} \operatorname{cdet}_{i}\left(\left(\mathbf{A}^{*} \mathbf{A}\right)_{. i}\left(\mathbf{a}_{. j}^{*}\right)\right)_{\beta}^{\beta}$ for all $k=1, \ldots, n-1$ and $c_{n}^{(i j)}=$ $\operatorname{cdet}_{i}\left(\mathbf{A}^{*} \mathbf{A}\right)_{. i}\left(\mathbf{a}_{. j}^{*}\right)$.

Now we prove that $c_{k}^{(i j)}=0$, when $k \geq r+1$ for all $i=1, \ldots, n$, and $j=1, \ldots, m$. By Lemma $4.1 \operatorname{rank}\left(\mathbf{A}^{*} \mathbf{A}\right)_{. i}\left(\mathbf{a}_{. j}^{*}\right) \leq r$, then the matrix $\left(\mathbf{A}^{*} \mathbf{A}\right)_{. i}\left(\mathbf{a}_{. j}^{*}\right)$ has no more $r$ right-linearly independent columns.

Consider $\left(\left(\mathbf{A}^{*} \mathbf{A}\right)_{. i}\left(\mathbf{a}_{. j}^{*}\right)\right)_{\beta}^{\beta}$, when $\beta \in J_{k, n}\{i\}$. It is a principal submatrix of $\left(\mathbf{A}^{*} \mathbf{A}\right)_{. i}\left(\mathbf{a}_{. j}^{*}\right)$ of order $k \geq r+1$. Deleting both its $i$-th row and column, we obtain a principal submatrix of order $k-1$ of $\mathbf{A}^{*} \mathbf{A}$. We denote it by $\mathbf{M}$. The following cases are possible.

Let $k=r+1$ and $\operatorname{det} \mathbf{M} \neq 0$. In this case all columns of $\mathbf{M}$ are right-linearly independent. The addition of all of them on one coordinate to columns of $\left(\left(\mathbf{A}^{*} \mathbf{A}\right)_{. i}\left(\mathbf{a}_{. j}^{*}\right)\right)_{\beta}^{\beta}$ keeps their right-linear independence. Hence, they are basis in a matrix $\left(\left(\mathbf{A}^{*} \mathbf{A}\right){ }_{. i}\left(\mathbf{a}_{. j}^{*}\right)\right)_{\beta}^{\beta}$, and by Theorem 2.7 the $i$-th column is the right linear combination of its basis columns. From this by Theorem [2.5, we get $\operatorname{cdet}_{i}\left(\left(\mathbf{A}^{*} \mathbf{A}\right)_{. i}\left(\mathbf{a}_{. j}^{*}\right)\right)_{\beta}^{\beta}=0$, when $\beta \in J_{k, n}\{i\}$ and $k \geq r+1$.

If $k=r+1$ and $\operatorname{det} \mathbf{M}=0$, then $p,(p<k)$, columns are basis in $\mathbf{M}$ and in $\left(\left(\mathbf{A}^{*} \mathbf{A}\right)_{. i}\left(\mathbf{a}_{. j}^{*}\right)\right)_{\beta}^{\beta}$. Then by Theorems 2.7 and 2.5 we obtain $\operatorname{cotet}_{i}\left(\left(\mathbf{A}^{*} \mathbf{A}\right)_{. i}\left(\mathbf{a}_{. j}^{*}\right)\right)_{\beta}^{\beta}=0$ as well.

If $k>r+1$, then from Theorems 2.6 and 2.8 it follows that $\operatorname{det} \mathbf{M}=$ 0 and $p,(p<k-1)$, columns are basis in the both matrices $\mathbf{M}$ and $\left(\left(\mathbf{A}^{*} \mathbf{A}\right)_{. i}\left(\mathbf{a}_{. j}^{*}\right)\right)_{\beta}^{\beta}$. Then by Theorems 2.7 and 2.5, we obtain that $\operatorname{cdet}_{i}\left(\left(\mathbf{A}^{*} \mathbf{A}\right)_{. i}\left(\mathbf{a}_{. j}^{*}\right)\right)_{\beta}^{\beta}=$ 0 .

Thus in all cases we have $\operatorname{cdet}_{i}\left(\left(\mathbf{A}^{*} \mathbf{A}\right)_{. i}\left(\mathbf{a}_{. j}^{*}\right)\right)_{\beta}^{\beta}=0$, when $\beta \in J_{k, n}\{i\}$ and $r+1 \leq k<n$. From here if $r+1 \leq k<n$, then $c_{k}^{(i j)}=\sum_{\beta \in J_{k, n}\{i\}} \operatorname{cdet}_{i}\left(\left(\mathbf{A}^{*} \mathbf{A}\right)_{. i}\left(\mathbf{a}_{. j}^{*}\right)\right)_{\beta}^{\beta}=$ 0 , and $c_{n}^{(i j)}=\operatorname{cdet}_{i}\left(\mathbf{A}^{*} \mathbf{A}\right)_{. i}\left(\mathbf{a}_{. j}^{*}\right)=0$ for all $i=1, \ldots, n$ and $j=1, \ldots, m$.

Hence, $\operatorname{cdet}_{i}\left(\alpha \mathbf{I}+\mathbf{A}^{*} \mathbf{A}\right)_{. i}\left(\mathbf{a}_{. j}^{*}\right)=c_{1}^{(i j)} \alpha^{n-1}+c_{2}^{(i j)} \alpha^{n-2}+\cdots+c_{r}^{(i j)} \alpha^{n-r}$ for all $i=1, \ldots, n$ and $j=1, \ldots, m$. By substituting these values in the matrix 
from (12), we obtain

$$
\begin{array}{r}
\mathbf{A}^{+}=\lim _{\alpha \rightarrow 0}\left(\begin{array}{lll}
\frac{c_{1}^{(11)} \alpha^{n-1}+\cdots+c_{r}^{(11)} \alpha^{n-r}}{\alpha^{n}+d_{1} \alpha^{n-1}+\cdots+d_{r} \alpha^{n-r}} & \ldots & \frac{c_{1}^{(1 m)} \alpha^{n-1}+\cdots+c_{r}^{(1 m)} \alpha^{n-r}}{\alpha^{n}+d_{1} \alpha^{n-1}+\cdots+d_{r} \alpha^{n-r}} \\
\ldots & \ldots & \ldots \\
\frac{c_{1}^{(n 1)} \alpha^{n-1}+\cdots+c_{r}^{(n 1)} \alpha^{n-r}}{\alpha^{n}+d_{1} \alpha^{n-1}+\cdots+d_{r} \alpha^{n-r}} & \ldots & \frac{c_{1}^{(n m)} \alpha^{n-1}+\cdots+c_{r}^{(n m)} \alpha^{n-r}}{\alpha^{n}+d_{1} \alpha^{n-1}+\cdots+d_{r} \alpha^{n-r}}
\end{array}\right)= \\
\left(\begin{array}{ccc}
\frac{c_{r}^{(11)}}{d_{r}} & \ldots & \frac{c_{r}^{(1 m)}}{d_{r}} \\
\ldots & \ldots & \ldots \\
\frac{c_{r}^{(n 1)}}{d_{r}} & \ldots & \frac{c_{r}^{(n m)}}{d_{r}}
\end{array}\right)
\end{array}
$$

Here $c_{r}^{(i j)}=\sum_{\beta \in J_{r, n}\{i\}} \operatorname{cdet}_{i}\left(\left(\mathbf{A}^{*} \mathbf{A}\right)_{. i}\left(\mathbf{a}_{. j}^{*}\right)\right)_{\beta}^{\beta}$ and $d_{r}=\sum_{\beta \in J_{r, n}}\left|\left(\mathbf{A}^{*} \mathbf{A}\right)_{\beta}^{\beta}\right|$. Thus, we have obtained the determinantal representation of $\mathbf{A}^{+}(10)$.

Similarly one can prove the determinantal representation of $\mathbf{A}^{+}$(11).

Remark 4.1 In (10) the index $i$ in $\operatorname{cdet}_{i}\left(\left(\mathbf{A}^{*} \mathbf{A}\right){ }_{. i}\left(\mathbf{a}_{. j}^{*}\right)\right)_{\beta}^{\beta}$ designates ith column of $\left(\left(\mathbf{A}^{*} \mathbf{A}\right){ }_{. i}\left(\mathbf{a}_{. j}^{*}\right)\right)$, but in the submatrix $\left(\left(\mathbf{A}^{*} \mathbf{A}\right)_{. i}\left(\mathbf{a}_{. j}^{*}\right)\right)_{\beta}^{\beta}$ the entries of $\mathbf{a}_{. j}^{*}$ may be placed in a column with the another index. In (11) we have equivalently.

Remark 4.2 If rank $\mathbf{A}=n$, then by Corollary $3.1 \mathbf{A}^{+}=\left(\mathbf{A}^{*} \mathbf{A}\right)^{-1} \mathbf{A}^{*}$. Considering $\left(\mathbf{A}^{*} \mathbf{A}\right)^{-1}$ as a left inverse, we get the following representation of $\mathbf{A}^{+}$:

$$
\mathbf{A}^{+}=\frac{1}{\operatorname{ddet} \mathbf{A}}\left(\begin{array}{ccc}
\operatorname{cdet}_{1}\left(\mathbf{A}^{*} \mathbf{A}\right)_{.1}\left(\mathbf{a}_{.1}^{*}\right) & \ldots & \operatorname{cdet}_{1}\left(\mathbf{A}^{*} \mathbf{A}\right)_{.1}\left(\mathbf{a}_{. m}^{*}\right) \\
\ldots & \ldots & \ldots \\
\operatorname{cdet}_{n}\left(\mathbf{A}^{*} \mathbf{A}\right)_{. n}\left(\mathbf{a}_{.1}^{*}\right) & \ldots & \operatorname{cdet}_{n}\left(\mathbf{A}^{*} \mathbf{A}\right)_{. n}\left(\mathbf{a}_{. m}^{*}\right)
\end{array}\right)
$$

If $m>n$, then by Theorem 4.1 for $\mathbf{A}^{+}$we have (10) as well.

Remark 4.3 If rank $\mathbf{A}=m$, then by Corollary 3.1 $\mathbf{A}^{+}=\mathbf{A}^{*}\left(\mathbf{A A}^{*}\right)^{-1}$. Considering $\left(\mathbf{A A}^{*}\right)^{-1}$ as a right inverse, we get the following representation of $\mathbf{A}^{+}$:

$$
\mathbf{A}^{+}=\frac{1}{\operatorname{ddet} \mathbf{A}}\left(\begin{array}{ccc}
\operatorname{rdet}_{1}\left(\mathbf{A A}^{*}\right)_{1 .}\left(\mathbf{a}_{1 .}^{*}\right) & \ldots & \operatorname{rdet}_{m}\left(\mathbf{A} \mathbf{A}^{*}\right)_{m .}\left(\mathbf{a}_{1 .}^{*}\right) \\
\ldots & \ldots & \ldots \\
\operatorname{rdet}_{1}\left(\mathbf{A A}^{*}\right)_{1 .}\left(\mathbf{a}_{n .}^{*}\right) & \ldots & \operatorname{rdet}_{m}\left(\mathbf{A A}^{*}\right)_{m .}\left(\mathbf{a}_{n .}^{*}\right)
\end{array}\right)
$$

If $m<n$, then by Theorem 4.1 for $\mathbf{A}^{+}$we also have (11). 
Corollary 4.1 If $\mathbf{A} \in \mathbb{H}_{r}^{m \times n}$, where $r<\min \{m, n\}$ or $r=m<n$, then for a projection matrix $\mathbf{A}^{+} \mathbf{A}=: \mathbf{P}=\left(p_{i j}\right)_{n \times n}$ we have its following determinantal representation

$$
p_{i j}=\frac{\sum_{\beta \in J_{r, n}\{i\}} \operatorname{cdet}_{i}\left(\left(\mathbf{A}^{*} \mathbf{A}\right)_{. i}\left(\mathbf{d}_{. j}\right)\right)_{\beta}^{\beta}}{\sum_{\beta \in J_{r, n}}\left|\left(\mathbf{A}^{*} \mathbf{A}\right)_{\beta}^{\beta}\right|},
$$

where $\mathbf{d}_{. j}$ is the $j$-th column of $\mathbf{A}^{*} \mathbf{A} \in \mathbb{H}^{n \times n}$ and for all $i, j=1, \ldots, n$.

Proof. Representing $\mathbf{A}^{+}$by (10) and right-multiplying it by $\mathbf{A}$, we obtain for an entry $p_{i j}$ of $\mathbf{A}^{+} \mathbf{A}=: \mathbf{P}=\left(p_{i j}\right)_{n \times n}$.

$$
\begin{gathered}
p_{i j}=\sum_{p=1}^{m} c_{i p} \cdot a_{p j}=\sum_{k} \frac{\sum_{\beta \in J_{r, n}\{i\}} \operatorname{cdet}_{i}\left(\left(\mathbf{A}^{*} \mathbf{A}\right)_{. i}\left(\mathbf{a}_{\cdot j}^{*}\right)\right)_{\beta}^{\beta}}{\sum_{\beta \in J_{r, n}}\left|\left(\mathbf{A}^{*} \mathbf{A}\right)_{\beta}^{\beta}\right|} \cdot a_{k j}= \\
=\frac{\sum_{\beta \in J_{r, n}\{i\}} \sum_{k} \operatorname{cdet}_{i}\left(\left(\mathbf{A}^{*} \mathbf{A}\right)_{. i}\left(\mathbf{a}_{. j}^{*}\right)\right)_{\beta}^{\beta} \cdot a_{k j}}{\sum_{\beta \in J_{r, n}}\left|\left(\mathbf{A}^{*} \mathbf{A}\right)_{\beta}^{\beta}\right|}=\frac{\sum_{\beta \in J_{r, n}\{i\}} \operatorname{det}_{i}\left(\left(\mathbf{A}^{*} \mathbf{A}\right)_{. i}\left(\mathbf{d}_{. j}\right)\right)_{\beta}^{\beta}}{\sum_{\beta \in J_{r, n}}\left|\left(\mathbf{A}^{*} \mathbf{A}\right)_{\beta}^{\beta}\right|},
\end{gathered}
$$

where $d_{. j}$ is the $j$ th column of $\mathbf{A}^{*} \mathbf{A} \in \mathbb{H}^{n \times n}$ and for all $i, j=1, \ldots, n$.

By analogy can be proved the following corollary.

Corollary 4.2 If $\mathbf{A} \in \mathbb{H}_{r}^{m \times n}$, where $r<\min \{m, n\}$ or $r=n<m$, then for the projection matrix $\mathbf{A A}^{+}=: \mathbf{Q}=\left(q_{i j}\right)_{m \times m}$ we have its following determinantal representation

$$
q_{i j}=\frac{\sum_{\alpha \in I_{r, m}\{i\}}\left|\left(\left(\mathbf{A} \mathbf{A}^{*}\right)_{i .}\left(\mathbf{g}_{j} .\right)\right)_{\alpha}^{\alpha}\right|}{\sum_{\alpha \in I_{r, m}}\left|\left(\mathbf{A} \mathbf{A}^{*}\right)_{\alpha}^{\alpha}\right|},
$$

where $\mathbf{g}_{j}$. is the $j$ th row of $\left(\mathbf{A A}^{*}\right) \in \mathbb{H}^{m \times m}$ and for all $i, j=1, \ldots, m$.

Remark 4.4 By definition of a classical adjoint matrix of $\mathbf{A} \in \mathbb{C}^{n \times n}$, Adj $[\mathbf{A}]$. $\mathbf{A}=\mathbf{A} \cdot \operatorname{Adj}[\mathbf{A}]=\operatorname{det} \mathbf{A} \cdot \mathbf{I}$. Let $\mathbf{A} \in \mathbb{H}^{m \times n}$. If $\operatorname{rank} \mathbf{A}=n$, the by Corollary 3.1 we have $\mathbf{A}^{+} \mathbf{A}=\mathbf{I}_{n}$. Representing $\mathbf{A}^{+}$by (13) as $\mathbf{A}^{+}=\frac{\mathbf{L}}{\operatorname{det}\left(\mathbf{A}^{*} \mathbf{A}\right)}$, where $\mathbf{L}=\left(\operatorname{cdet}_{i}\left(\left(\mathbf{A}^{*} \mathbf{A}\right)_{. i}\left(\mathbf{a}_{. j}^{*}\right)\right)\right)_{n \times m}$, we obtain $\mathbf{L A}=\operatorname{det}\left(\mathbf{A}^{*} \mathbf{A}\right) \cdot \mathbf{I}_{n}$. This means that the matrix $\mathbf{L}=: \operatorname{Adj}_{L}[\mathbf{A}]$ is the left classical adjoint matrix of $\mathbf{A} \in \mathbb{H}^{m \times n}$. 
If $\operatorname{rank} \mathbf{A}=m$, then by definition of a right classical adjoint matrix of $\mathbf{A} \in \mathbb{H}^{m \times n}$ by Corollary 3.1 and by (14) we can put

$$
\operatorname{Adj}_{R}[\mathbf{A}]:=\left(\left(\operatorname{rdet}_{j}\left(\mathbf{A A}^{*}\right)_{j .}\left(\mathbf{a}_{i .}^{*}\right)\right)\right)_{m \times n},
$$

since in this case $\mathbf{A} \cdot \operatorname{Adj}_{R}[\mathbf{A}]=\operatorname{det}\left(\mathbf{A} \mathbf{A}^{*}\right) \cdot \mathbf{I}$.

If $\operatorname{rank} \mathbf{A}=r<\min \{m, n\}$, then an analog of a left classical adjoint matrix of $\mathbf{A} \in \mathbb{H}^{m \times n}$ by (10) can accept

$$
\operatorname{Adj}_{L}[\mathbf{A}]:=\left(\sum_{\alpha \in J_{r, n}\{i\}} \operatorname{cdet}_{i}\left(\left(\mathbf{A}^{*} \mathbf{A}\right)_{. i} \mathbf{a}_{. j}^{*}\right)_{\alpha}^{\alpha}\right)_{n \times m} .
$$

Indeed, since eigenvalues of a projection matrix are only 1 and 0 , there exists such a unitary matrix $\mathbf{U} \in \mathbb{H}^{n \times n}$ that

$$
\begin{gathered}
\operatorname{Adj}_{L}[\mathbf{A}] \cdot \mathbf{A}=\sum_{\alpha \in I_{r, n}}\left|\left(\mathbf{A}^{*} \mathbf{A}\right)_{\alpha}^{\alpha}\right| \cdot \mathbf{P}= \\
=\sum_{\alpha \in I_{r, n}}\left|\left(\mathbf{A}^{*} \mathbf{A}\right)_{\alpha}^{\alpha}\right| \cdot \mathbf{U d i a g}(1, \ldots, 1,0, \ldots, 0) \mathbf{U}^{*} .
\end{gathered}
$$

If $\operatorname{rank} \mathbf{A}=r<\min \{m, n\}$, then by an analogue of a right classical adjoint matrix of $\mathbf{A} \in \mathbb{H}^{m \times n}$ by (11) we can put

$$
\operatorname{Adj}_{R}[\mathbf{A}]:=\left(\sum_{\alpha \in I_{r, m}\{j\}} \operatorname{rdet}_{j}\left(\left(\mathbf{A A}^{*}\right)_{j}\left(\mathbf{a}_{i .}^{*}\right)\right)_{\alpha}^{\alpha}\right)_{n \times m},
$$

as there exists such a unitary matrix $\mathbf{V} \in \mathbb{H}^{m \times m}$ that

$$
\begin{gathered}
\mathbf{A} \cdot \operatorname{Adj}_{R}[\mathbf{A}]=\sum_{\alpha \in J_{r, m}}\left|\left(\mathbf{A A}^{*}\right)_{\alpha}^{\alpha}\right| \cdot \mathbf{Q}= \\
=\sum_{\alpha \in J_{r, m}}\left|\left(\mathbf{A A}^{*}\right)_{\alpha}^{\alpha}\right| \cdot \mathbf{V d i a g}(1, \ldots, 1,0, \ldots, 0) \mathbf{V}^{*} .
\end{gathered}
$$

Remark 4.5 If $\mathbf{A} \in \mathbb{C}^{m \times n}$ is a matrix with complex entries, then we obtain the following analogs of (10) and (11), respectively,

$$
a_{i j}^{+}=\frac{\sum_{\beta \in J_{r, n}\{i\}}\left|\left(\left(\mathbf{A}^{*} \mathbf{A}\right)_{. i}\left(\mathbf{a}_{. j}^{*}\right)\right)_{\beta}^{\beta}\right|}{\sum_{\beta \in J_{r, n}}\left|\left(\mathbf{A}^{*} \mathbf{A}\right)_{\beta}^{\beta}\right|}, \quad a_{i j}^{+}=\frac{\sum_{\alpha \in I_{r, m}\{j\}}\left|\left(\left(\mathbf{A} \mathbf{A}^{*}\right)_{j .}\left(\mathbf{a}_{i .}^{*}\right)\right)_{\alpha}^{\alpha}\right|}{\sum_{\alpha \in I_{r, m}}\left|\left(\mathbf{A} \mathbf{A}^{*}\right)_{\alpha}^{\alpha}\right|}
$$


for all $i=1, \ldots, n$ and $j=1, \ldots, m$. These determinantal representations are original in this case as well. It is reflected in [12]. The analogous result is obtained in [20].

Remark 4.6 The Gram polynomial of a matrix $\mathbf{A}$,

$$
\operatorname{det}\left(\mathbf{I}_{m}+z \mathbf{A A}^{*}\right)=1+a_{1} z+\cdots+a_{m} z^{m}
$$

is used for generalizing the Moore-Penrose inverse and the classical Cramer's rule to the corresponding undetermined and overdetermined cases over an arbitrary field in [8] as well.

\section{$5 \quad$ Cramer's rule for a least squares solution of quaternion system linear equations}

Definition 5.1 Suppose

$$
\mathbf{A} \cdot \mathbf{x}=\mathbf{y}
$$

is a right system linear equations over the quaternion skew field $\mathbb{H}$, where $\mathbf{A} \in \mathbb{H}^{m \times n}$ is the coefficient matrix, $\mathbf{y} \in \mathbb{H}^{m \times 1}$ is a column of constants, and $\mathbf{x} \in \mathbb{H}^{n \times 1}$ is a unknown column. The least square solution of (15) (with the least norm) is called the vector $\mathbf{x}^{0}$ satisfying

$$
\left\|\mathbf{x}^{0}\right\|=\min _{\mathbf{x} \in \mathbb{H}^{n}}\left\{\|\tilde{\mathbf{x}}\|:\|\mathbf{A} \cdot \tilde{\mathbf{x}}-\mathbf{y}\|=\min _{\mathbf{x} \in \mathbb{H}^{n}}\|\mathbf{A} \cdot \mathbf{x}-\mathbf{y}\|\right\},
$$

where $\mathbb{H}^{n}$ is an n-dimension right quaternion vector space.

We recall that in the right quaternion vector space $\mathbb{H}^{n}$ by definition of the inner product of vectors we put $\langle\mathbf{x}, \mathbf{y}\rangle:=\mathbf{y}^{*} \mathbf{x}=\overline{y_{1}} \cdot x_{1}+\cdots+\overline{y_{n}} \cdot x_{n}$ and $\|\mathbf{x}\|:=\sqrt{\langle\mathbf{x}, \mathbf{x}\rangle}$ is the norm of a vector $\mathbf{x} \in \mathbb{H}^{n}$. By analogy to a complex case (see, e.g. [7]) we can prove the following theorem.

Theorem 5.1 The vector $\mathbf{x}=\mathbf{A}^{+} \mathbf{y}$ is the least square solution of (15).

Definition 5.2 Suppose

$$
\mathbf{x} \cdot \mathbf{A}=\mathbf{y}
$$

is a left system linear equations over the quaternion skew field $\mathbb{H}$, where $\mathbf{A} \in \mathbb{H}^{m \times n}$ is the coefficient matrix, $\mathbf{y} \in \mathbb{H}^{1 \times n}$ is a row of constants, and 
$\mathbf{x} \in \mathbb{H}^{1 \times m}$ is a unknown row. The least square solution of (16) (with the least norm) is called the vector $\mathbf{x}^{0}$ satisfying

$$
\left\|\mathbf{x}^{0}\right\|=\min _{\tilde{\mathbf{x}} \in m \mathbb{H}}\left\{\|\tilde{\mathbf{x}}\|:\|\tilde{\mathbf{x}} \cdot \mathbf{A}-\mathbf{y}\|=\min _{\mathbf{x} \in \mathbb{m}_{\mathbb{H}}}\|\mathbf{x} \cdot \mathbf{A}-\mathbf{y}\|\right\},
$$

where $^{m} \mathbb{H}$ is an $m$-dimension left quaternion vector space.

We recall that in the left quaternion vector space ${ }^{m} \mathbb{H}$ by definition of the inner product of vectors we can put $\langle\mathbf{x}, \mathbf{y}\rangle=\mathbf{x y}^{*}=x_{1} \cdot \overline{y_{1}}+\cdots+x_{m} \cdot \overline{y_{m}}$. Then $\|\mathbf{x}\|:=\sqrt{\langle\mathbf{x}, \mathbf{x}\rangle}$ is the norm of $\mathbf{x} \in{ }^{m} \mathbb{H}$.

Theorem 5.2 The vector $\mathbf{x}=\mathbf{y} \cdot \mathbf{A}^{+}$is the least square solution of (16).

Theorem 5.3 (i) If $\operatorname{rank} \mathbf{A}=n$, then for the least square solution $\mathbf{x}^{0}=$ $\left(x_{1}^{0}, \ldots, x_{n}^{0}\right)^{T}$ of (15), we get for all $j=1, \ldots, n$

$$
x_{j}^{0}=\frac{\operatorname{cdet}_{j}\left(\mathbf{A}^{*} \mathbf{A}\right)_{. j}(\mathbf{f})}{\operatorname{ddet} \mathbf{A}},
$$

where $\mathbf{f}=\mathbf{A}^{*} \mathbf{y}$.

(ii) If $\operatorname{rank} \mathbf{A}=k \leq m<n$, then for all $j=1, \ldots, n$ we have

$$
x_{j}^{0}=\frac{\sum_{\beta \in J_{r, n}\{j\}} \operatorname{cdet}_{j}\left(\left(\mathbf{A}^{*} \mathbf{A}\right)_{. j}(\mathbf{f})\right)_{\beta}^{\beta}}{\sum_{\beta \in J_{r, n}}\left|\left(\mathbf{A}^{*} \mathbf{A}\right)_{\beta}^{\beta}\right|} .
$$

Proof. i) If rank $\mathbf{A}=n$, then $\mathbf{A}^{+}$can be represented by (13). Denote $\mathbf{f}:=\mathbf{A}^{*} \mathbf{y}$. Representing $\mathbf{A}^{+} \mathbf{y}$ by coordinates we obtain (17).

ii) If $\operatorname{rank} \mathbf{A}=k \leq m<n$, then by Theorem 4.1 we represent the matrix $\mathbf{A}^{+}$by (10). Representing $\mathbf{A}^{+} \mathbf{y}$ by coordinates we obtain (18).

Remark 5.1 In a complex case the following analogs of (17) and (18) are obtained respectively in [12] for all $j=1, \ldots, n$,

$$
x_{j}^{0}=\frac{\operatorname{det}\left(\mathbf{A}^{*} \mathbf{A}\right)_{. j}(\mathbf{f})}{\operatorname{det}\left(\mathbf{A}^{*} \mathbf{A}\right)}, x_{j}^{0}=\frac{\sum_{\beta \in J_{r, n}\{j\}}\left|\left(\left(\mathbf{A}^{*} \mathbf{A}\right)_{. j}(\mathbf{f})\right)_{\beta}^{\beta}\right|}{\sum_{\beta \in J_{r, n}}\left|\left(\mathbf{A}^{*} \mathbf{A}\right)_{\beta}^{\beta}\right|} .
$$


Theorem 5.4 (i) If $\operatorname{rank} \mathbf{A}=m$, then for $\mathbf{x}^{0}=\left(x_{1}^{0}, \ldots, x_{m}^{0}\right)$ of (16) we obtain for all $i=1, \ldots, m$

$$
x_{i}^{0}=\frac{\operatorname{rdet}_{i}\left(\mathbf{A} \mathbf{A}^{*}\right)_{i .}(\mathbf{z})}{\operatorname{ddet} \mathbf{A}}
$$

where $\mathbf{z}=\mathbf{y A}^{*}$.

(ii) If rank $\mathbf{A}=k \leq n<m$, then for all $i=1, \ldots, m$ we have

$$
x_{i}^{0}=\frac{\sum_{\alpha \in I_{r, m}\{i\}} \operatorname{rdet}_{i}\left(\left(\mathbf{A A}^{*}\right)_{i .}(\mathbf{z})\right)_{\alpha}^{\alpha}}{\sum_{\alpha \in I_{r, m}}\left|\left(\mathbf{A} \mathbf{A}^{*}\right)_{\alpha}^{\alpha}\right|} .
$$

The proof of this theorem is analogous to that of Theorem 5.3 .

Remark 5.2 In a complex case the following analogs of (19) and (20) respectively are obtained in [12] for all $i=1, \ldots, m$,

$$
x_{i}^{0}=\frac{\operatorname{det}\left(\mathbf{A A}^{*}\right)_{i .}(\mathbf{z})}{\operatorname{det} \mathbf{A} \mathbf{A}^{*}}, \quad x_{i}^{0}=\frac{\sum_{\alpha \in I_{r, m}\{i\}}\left|\left(\left(\mathbf{A} \mathbf{A}^{*}\right)_{i .}(\mathbf{z})\right)_{\alpha}^{\alpha}\right|}{\sum_{\alpha \in I_{r, m}}\left|\left(\mathbf{A} \mathbf{A}^{*}\right)_{\alpha}^{\alpha}\right|} .
$$

\section{Example}

Let us consider the left system of linear equations.

$$
\left\{\begin{array}{l}
x_{1} i+2 x_{2} i-x_{3}=i \\
-x_{1} k+x_{2} j+x_{3} j=j \\
x_{1} j+x_{2}+x_{3} k=k \\
x_{1}+x_{2} k+x_{3} i=1
\end{array}\right.
$$

The coefficient matrix of the system is the matrix $\mathbf{A}=\left(\begin{array}{cccc}i & -k & j & 1 \\ 2 i & j & 1 & k \\ -1 & j & k & i\end{array}\right)$.

The row of unknown is $\mathbf{x}=\left(\begin{array}{lll}x_{1} & x_{2} & x_{3}\end{array}\right)$ and the row of constants is $\mathbf{y}=\left(\begin{array}{llll}i & j & k & 1\end{array}\right)$. Then for (21) we have $\mathbf{x} \cdot \mathbf{A}=\mathbf{y}$. We obtain

$$
\mathbf{A}^{*}=\left(\begin{array}{ccc}
-i & -2 i & -1 \\
k & -j & -j \\
-j & 1 & -k \\
1 & -k & -i
\end{array}\right)
$$




$$
\mathbf{A A}^{*}=\left(\begin{array}{ccc}
4 & 2-i+j-k & -4 i \\
2+i-j+k & 7 & 1-2 i-j-k \\
4 i & 1+2 i+j+k & 4
\end{array}\right) .
$$

Since $\operatorname{ddet} \mathbf{A}=\operatorname{det} \mathbf{A} \mathbf{A}^{*}=\operatorname{rdet}_{1} \mathbf{A} \mathbf{A}^{*}=0$ and

$$
\begin{gathered}
\operatorname{det}\left(\mathbf{A A}^{*}\right)^{33}=\operatorname{rdet}_{1}\left(\begin{array}{cc}
4 & 2-i+j-k \\
2+i-j+k & 7
\end{array}\right)= \\
=4 \cdot 7-(2-i+j-k) \cdot(2+i-j+k)=21 \neq 0,
\end{gathered}
$$

by Lemma 2.6 $\operatorname{rank} \mathbf{A}=2$. We shall represent $\mathbf{A}^{+}$by (11).

$$
\begin{gathered}
\sum_{\alpha \in I_{2,3}}\left|\left(\mathbf{A A}^{*}\right)_{\alpha}^{\alpha}\right|=\operatorname{det}\left(\begin{array}{cc}
4 & 2-i+j-k \\
2+i-j+k & 7
\end{array}\right)+ \\
+\operatorname{det}\left(\begin{array}{cc}
7 & 1-2 i-j-k \\
1+2 i+j+k & 4
\end{array}\right)+\operatorname{det}\left(\begin{array}{cc}
4 & -4 i \\
4 i & 4
\end{array}\right)=42 .
\end{gathered}
$$

Now we shall calculate $r_{i j}=\sum_{\alpha \in I_{2,3}\{j\}} \operatorname{rdet}_{j}\left(\left(\mathbf{A A}^{*}\right)_{j} .\left(\mathbf{a}_{i .}^{*}\right)\right)_{\alpha}^{\alpha}$ for all $i=\overline{1,4}$ and $j=\overline{1,3}$. To obtain $r_{11}$, we consider the matrix

$$
\left(\mathbf{A A}^{*}\right)_{1 .}\left(\mathbf{a}_{1 .}^{*}\right)=\left(\begin{array}{ccc}
-i & -2 i & -1 \\
2+i-j+k & 7 & 1-2 i-j-k \\
4 i & 1+2 i+j+k & 4
\end{array}\right)
$$

Then we have

$$
\begin{gathered}
r_{11}=\operatorname{rdet}_{1}\left(\begin{array}{cc}
-i & -2 i \\
2+i-j+k & 7
\end{array}\right)+\operatorname{rdet}_{1}\left(\begin{array}{cc}
-i & -1 \\
4 i & 4
\end{array}\right)= \\
=-i \cdot 7-(-2 i) \cdot(2+i-j+k)-i \cdot 4-(-1 \cdot 4 i)=-2-3 i-2 j-2 k,
\end{gathered}
$$

and so forth. Continuing in the same way, we get

$$
\mathbf{A}^{+}=\frac{1}{42}\left(\begin{array}{ccc}
-2-3 i-2 j-2 k & 2-12 i+2 j+2 k & -3+2 i+2 j-2 k \\
1+i+2 j+6 k & -2+2 i-6 j-4 k & 1-i-6 j+2 k \\
-2-i-6 j-k & 6-2 i+4 j+2 k & -1+2 i+j-6 k \\
6+i+j+2 k & -4+2 i-2 j-6 k & 1-6 i-2 j+k
\end{array}\right)
$$

We find the least square solution by means of the matrix method by Theorem 5.2

$$
\mathbf{x}^{0}=\mathbf{y} \cdot \mathbf{A}^{+}=\frac{1}{42}(8+11 i+3 j-3 k, \quad 12-4 i-8 j, \quad 11-8 i+3 j+3 k) .
$$


Now we shall find the least square solution of (21) by means of Cramer's rule by (20). We have $\mathbf{z}=\mathbf{y} \cdot \mathbf{A}^{*}=(2+2 i, 3,2-2 i)$. Since

$$
\left(\mathbf{A A}^{*}\right)_{1 .}(\mathbf{z})=\left(\begin{array}{ccc}
2+2 i & 3 & 2-2 i \\
2+i-j+k & 7 & 1-2 i-j-k \\
4 i & 1+2 i+j+k & 4
\end{array}\right),
$$

we get

$$
\begin{aligned}
x_{1}^{0}= & \frac{\operatorname{rdet}_{1}\left(\begin{array}{cc}
2+2 i & 3 \\
2+i-j+k & 7
\end{array}\right)+\operatorname{rdet}_{1}\left(\begin{array}{cc}
2+2 i & 2-2 i \\
4 i & 4
\end{array}\right)}{\sum_{\alpha \in I_{2,3}}\left|\left(\mathbf{A A} \mathbf{A}^{*}\right)_{\alpha}^{\alpha}\right|}=\frac{8+11 i+3 j-3 k}{42} . \\
& \text { Since }\left(\mathbf{A A}^{*}\right)_{2 .}(\mathbf{z})=\left(\begin{array}{ccc}
4 & 2-i+j-k & -4 i \\
2+2 i & 3 & 2-2 i \\
4 i & 1+2 i+j+k & 4
\end{array}\right), \\
x_{2}^{0}= & \frac{\operatorname{rdet}_{2}\left(\begin{array}{ccc}
4 & 2-i+j-k \\
2+2 i & 3
\end{array}\right)+\operatorname{rdet}_{1}\left(\begin{array}{cc}
3 & 2-2 i \\
1+2 i+j+k & 4
\end{array}\right)}{\sum_{\alpha \in I_{2,3}}\left|\left(\mathbf{A A}^{*}\right)_{\alpha}^{\alpha}\right|}=\frac{12-4 i-8 j}{42} .
\end{aligned}
$$

Since

$$
\begin{gathered}
\left(\mathbf{A A}^{*}\right)_{3} .(\mathbf{z})=\left(\begin{array}{ccc}
4 & 2-i+j-k & -4 i \\
2+i-j+k & 7 & 1-2 i-j-k \\
2+2 i & 3 & 2-2 i
\end{array}\right), \\
x_{3}^{0}=\frac{\operatorname{rdet}_{2}\left(\begin{array}{cc}
4 & -4 i \\
2+2 i & 2-2 i
\end{array}\right)+\operatorname{rdet}_{1}\left(\begin{array}{cc}
7 & 1-2 i-j-k \\
3 & 2-2 i
\end{array}\right)}{\sum_{\alpha \in I_{2,3}}\left|\left(\mathbf{A A}^{*}\right)_{\alpha}^{\alpha}\right|}=\frac{11-8 i+3 j+3 k}{42} .
\end{gathered}
$$

\section{References}

[1] H. Aslaksen, Quaternionic determinants, Math. Intelligencer 18(1996), no.3, pp. 57-65.

[2] A. Baker, Right eigenvalues for quaternionic matrices: a topological approach, Linear Algebra and its Applications 286 (1999), pp. 303-309. 
[3] D. Carl, C.D. Meyer Jr., Limits and the index of a square matrix, SIAM J. Appl. Math. 26 (1974), no.3, pp. 506-515.

[4] L. Chen, Definition of determinant and Cramer solutions over quaternion field, Acta Math. Sinica (N.S.) 7 (1991), pp. 171-180.

[5] J. Diedonne, Les determinantes sur une corps non commutatif, Bull. Soc. Math. France 71 (1943), pp. 27-45.

[6] T. Dray, C. A. Manogue, The octonionic eigenvalue problem, Advances in Applied Clifford Algebras 8 (1998), no. 2, pp. 341-364.

[7] F. R. Gantmacher, The theory of matrices, Trans. from the Russian by K. A. Hirsch, vols. I and II. New York, Chelsea, 1959.

[8] Gema M. Diaz-Roca, Laureano Gonzalez-Vega, Henri Lombardi, Generalizing Cramers rule: solving uniformly linear systems of equations, SIAM J. Matrix Anal. Appl. Vol. 27 (2005), no. 3, pp. 621-637.

[9] L. Huang, W. So, On left eigenvalues of a quaternionic matrix, Linear Algebra Appl. 323 (2001), pp. 105-116.

[10] T. Jiang, L. Chen, Algebraic algorithms for least squares problem in quaternionic quantum theory, Comp. Phys. Communications 176 (2007), pp. 481-485.

[11] I. I. Kyrchei, Cramer's rule for quaternionic systems of linear equations, Journal of Mathematical Sciences 155 (2008), no 6, pp. 839-858. Translated from Fundamental and Appl. Math. 13 (2007), no.4, pp. 67-94. (Russian) arXiv:math/0702447v1 [math.RA]

[12] I. I. Kyrchei, Analogs of the adjoint matrix for generalized inverses and corresponding Cramer rules, Linear and Multilinear algebra 56 (2008) no.4, pp. 453-469. arXiv:1004.4761v1 [math.RA]

[13] P. Lancaster, M. Tismenitsky, Theory of matrices, Acad. Press., New York 1969.

[14] E. H. Moore, On reciprocal of the general algebraic matrix, Bull. Amer. Math. Soc. 26 (1920), pp. 394-395. 
[15] W. So, Quaternionic left eigenvalue problem, Southeast Asian Bulletin of Mathematics, 29 (2005), pp. 555-565.

[16] E. Study, Zur Theorie der linearen Gleichungen, Acta Math. 42 (1920), pp. 1-61.

[17] N. A. Wiegmann, Some theorems on matrices with real quaternion elements, Canad. J. Math. 7 (1955), pp. 191-201.

[18] R. M. W. Wood, Quaternionic eigenvalues, Bull. Lond. Math. Soc. 17 (1985), pp. 137-138.

[19] Z. Yang, The covariance condition of the Moore-Penrose inverse of a quternion matrix, Northeast. Math. J. 5 (1989), no.3, pp. 277-282.

[20] Y. Yu, Y. Wei, Determinantal representation of the generalized inverse $A_{T, S}^{(2)}$ over integral domains and its applications, Linear and Multilinear algebra 57 (2009) no.6, pp. 547-559.

[21] F. Zhang, Quaternions and matrices of quaternions, Linear Algebra Appl. 251 (1997), pp. 21-57.

[22] W. Zhuang, Involutary functions and generalized inverses matrices over an arbitrary skew field, Northeast. Math. J. 3 (1987), no.1, pp. 57-66. 